\title{
Quantitative Proteomic Analyses Identify STO/BBX24 -Related Proteins Induced by UV-B
}

\author{
Guizhen Lyu ${ }^{1,+} \mathbb{C}$, Dongbing Li ${ }^{1,+} \mathbb{C}$, Hui Xiong ${ }^{1}$, Langtao Xiao ${ }^{2}$, Jianhua Tong ${ }^{2}$, \\ Chanjuan Ning ${ }^{1}$, Ping Wang ${ }^{1}$ and Shaoshan $\mathrm{Li}^{1, *(D)}$ \\ 1 Key Laboratory of Ecology and Environmental Science in Guangdong Higher Education, \\ School of Life Science, South China Normal University, Guangzhou 510631, China; \\ christinzhen@163.com (G.L.); ldb06138233@163.com (D.L.); xionghui_1995@163.com (H.X.); \\ ncj1983@163.com (C.N.); wangping@beijingtest.com (P.W.) \\ 2 Hunan Provincial Key Laboratory of Phytohormones, Hunan Agricultural University, \\ Changsha 410128, China; langtaoxiao@163.com (L.X.); tjh0421@sohu.com (J.T.) \\ * Correspondence: lishsh@scnu.edu.cn; Tel.: +86-020-85211555 \\ + These authors contributed equally to this work.
}

Received: 26 February 2020; Accepted: 31 March 2020; Published: 3 April 2020

\begin{abstract}
Plants use solar radiation for photosynthesis and are inevitably exposed to UV-B. To adapt to UV-B radiation, plants have evolved a sophisticated strategy, but the mechanism is not well understood. We have previously reported that STO (salt tolerance)/BBX24 is a negative regulator of UV-B-induced photomorphogenesis. However, there is limited knowledge of the regulatory network of STO in UV-B signaling. Here, we report the identification of proteins differentially expressed in the wild type (WT) and sto mutant after UV-B radiation by iTRAQ (isobaric tags for relative and absolute quantitation)-based proteomic analysis to explore differential proteins that depend on STO and UV-B signaling. A total of 8212 proteins were successfully identified, 221 of them were STO-dependent proteins in UV-B irradiated plants. The abundances of STO-dependent PSB and LHC (light-harvesting complex) proteins in sto mutants decreased under UV-B radiation, suggesting that STO is necessary to maintain the normal accumulation of photosynthetic system complex under UV-B radiation to facilitate photosynthesis photon capture. The abundance of phenylalanine lyase-1 (PAL1), chalcone synthetase (CHS), and flavonoid synthetase (FLS) increased significantly after UV-B irradiation, suggesting that the accumulation of flavonoids do not require STO, but UV-B is needed. Under UV-B radiation, STO stabilizes the structure of antenna protein complex by maintaining the accumulation of PSBs and LHCs, thereby enhancing the non-photochemical quenching (NPQ) ability, releasing extra energy, protecting photosynthesis, and ultimately promoting the elongation of hypocotyl. The accumulation of flavonoid synthesis key proteins is independent of STO under UV-B radiation. Overall, our results provide a comprehensive regulatory network of STO in UV-B signaling.
\end{abstract}

Keywords: UV-B irradiation; STO (salt tolerance)/BBX24; photosynthesis; antenna protein; flavanol; anthocyanin; NPQ (non-photochemical quenching)

\section{Introduction}

Plants grown in the natural environment are inevitably affected by UV-B. While animals can actively move to avoid UV radiation, plants have to adapt to UV-B radiation by synthesizing sunscreen substances such as flavonoids and anthocyanin [1,2]. High UV-B radiation induces stress responses in plants, such as damage to DNA, proteins, and the plasma membrane, accumulation of reactive oxygen species, cell cycle arrest, chlorophyll degradation, and inhibition of photosynthesis [3-5]. In recent years, UV-B has been revealed as a stress factor that alters gene expression related to physiological processes 
such as metabolism, morphogenesis, and photosynthesis [6]. UV-B also affects leaf development, circadian rhythm, and flowering time [7].

Previous biochemical assays identified UVR8 (UV resistance locus 8) as a UV-B receptor [8] and resolved its high-resolution structure [9]. UVR8 has a high sequence similarity with RCC1 (regulator of chromatin condensation 1) in humans and is highly conserved in plants [10]. In Arabidopsis, several protein complexes that receive and transmit UV-B signals have been investigated, including UVR8-COP1 (constitutively photomorphogenic 1), UVR8-WRKY36 (WRKY DNA-binding protein 36), and UVR8-BES1 (BRI1-EMS-suppressor1)/BIM1 (BES1-interacting MYC-like 1) [11]. The UV-B-induced transcription factor HY5 (elongated hypocotyl 5) is mediated by two major ways to activate downstream genes: (1) HY5 is synthesized in the presence of the nuclear UVR8-COP1 complex and then activates HY5 [1,2,12]; (2) in the nucleus, the UVR8-WRKY36 complex prevents WRKY36 (a repressor of HY5) from binding to HY5 promoter [13]. In addition, the UVR8-BIM1 complex dephosphorylates BES1, thus inhibiting the induction of BR-responsive genes related to hypocotyl elongation and plant growth by disrupting the formation of the BIM1-BES complex [14].

Our previous studies demonstrate the effect of salt tolerance (STO) on UV-B signaling. UV-B induces both the transcripts and protein levels of STO, and the sto mutants display defective hypocotyl elongation compared to the WT upon UV-B radiation. STO interacts with COP1 (an ubiquitin E3 ubiquitin ligase and a central mediator of UV-B signaling) in a UV-B-dependent manner. STO also suppresses HY5 transcription. In addition, STO interacts with RCD1 in vitro. Our genetic analysis reveal that the $r c d 1-1$ and sto mutant have similar hypocotyl length upon UV-B radiation, while 35S-STO-GFP overexpression could rescue the long hypocotyl phenotype of $r c d 1-1$ [15]. Moreover, STO is involved in other biological processes including salt stress [16], red and far-red light [17], circadian rhythm and flowering [3], and seedling development [18]. Hypocotyls of the $b b \times 24 b b \times 25$ mutant become shorter [19].

Recently, our research confirmed STO and GA negatively regulate UV-B-induced Arabidopsis root growth inhibition [20]. However, the mechanism by which STO regulates UV-B signaling is still unknown. Therefore, iTRAQ-based proteomics technology was used to investigate the mechanism of STO under UV-B radiation in the present research. Screening the STO-dependent UV-B-induced proteins lays a solid foundation for the later study of STO response to complex climate change.

\section{Results}

\subsection{General Characteristics of STO-Dependent UV-B-Induecd Proteins}

To reveal the regulatory network of STO in UV-B signaling, we extracted total proteins from the sto mutant and WT (Col-0) for proteome analysis using iTRAQ. Ultimately, 8212 proteins were identified. We listed the detailed information of each protein in Table 1.

Table 1. Information about the proteins

\begin{tabular}{ccccccc}
\hline Database & NO. & $\begin{array}{c}\text { Total } \\
\text { Spectra }\end{array}$ & $\begin{array}{c}\text { Peptide Spectrum } \\
\text { Match }\end{array}$ & Peptides & $\begin{array}{c}\text { Unique } \\
\text { Peptides }\end{array}$ & $\begin{array}{c}\text { Protein } \\
\text { Groups }\end{array}$ \\
\hline TAIR & 1 & 312,550 & 94,209 & 41,480 & 36,822 & 7306 \\
TAIR & 2 & 307,230 & 94,057 & 40,076 & 35,436 & 7130 \\
TAIR & 3 & 306,008 & 93,046 & 38,283 & 33,967 & 6931 \\
TAIR & Total & 925,788 & 281,312 & 54,992 & 48,791 & 8212 \\
\hline
\end{tabular}

Among the identified proteins, 41 differentially expressed proteins were from (+)WT/(-)WT, of which 14 were down-regulated regulated (fold change $<0.83, P<0.05$ ) and 27 were up-regulated (fold change $>1.2, P<0.05$ ). Three hundred and twenty-two differentially expressed proteins were from the UV-B-exposed sto mutant, of which 213 were up-regulated and 109 were down-regulated. A total of 614 differentially expressed proteins were from $(+)$ sto/(+)WT, of which 292 were up-regulated and 322 were down-regulated (Table 2). Based on the abundance of the differentially expressed proteins 
(DEPs), a hierarchical clustering analysis was performed (Figure 1). The biological replicates showed good repeatability within each treatment group, but data between different treatment groups displayed low similarity. Moreover, according to the clustering analysis, the variation in protein abundance indicated the high frequency and complexity of differential protein expression in sto mutant after UV-B treatment (Figure 1B). The clustering analysis indicates that STO mediates a large portion of the differential protein expression in response to UV-B.

Table 2. Number of differentially expressed proteins in each comparison group.

\begin{tabular}{cccc}
\hline Comparison Group & Up-Regulated & Down-Regulated & In Total \\
\hline (+)WT/(-)WT & 27 & 14 & 41 \\
(+)sto/(-)sto & 213 & 109 & 322 \\
(+)sto/(+)WT & 292 & 322 & 614 \\
(-)sto/(-)WT & 45 & 39 & 84
\end{tabular}

Note: Fold change more than 1.2 times is up-regulated, and less than 0.83 times is down-regulated, $P<0.05$.

A

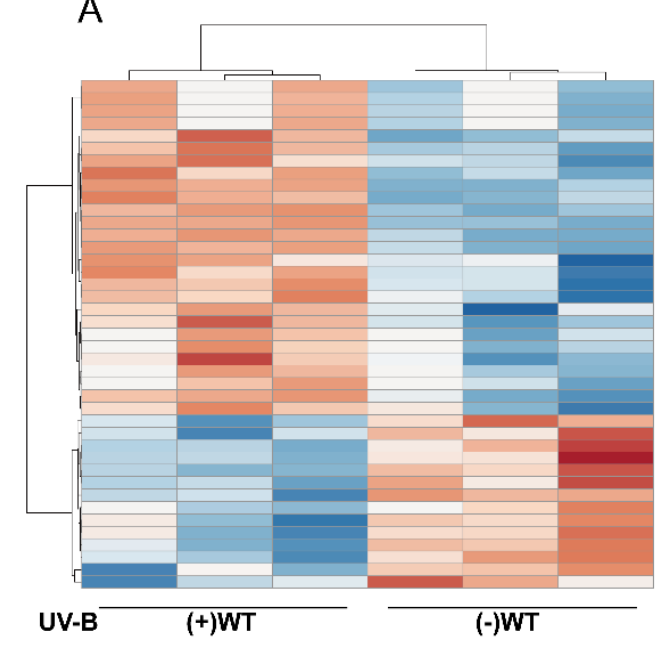

B

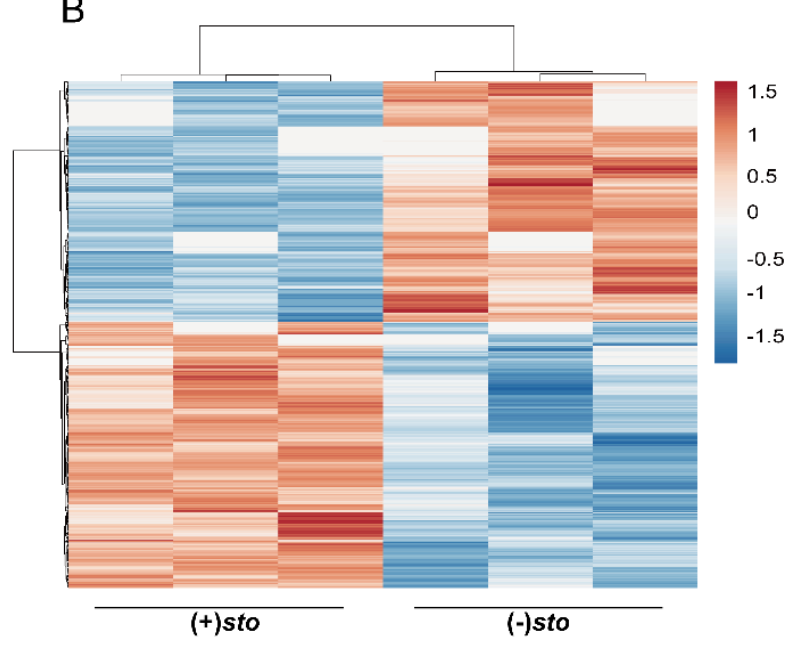

Figure 1. Clustering analysis of the differentially expressed proteins. (A) A hierarchical clustering analysis of the differentially expressed proteins in the wild-type (WT) seedlings with (+) or without (-) UV-B radiation. (B) A hierarchical clustering analysis of the differentially expressed proteins in the sto seedlings with (+) or without (-) UV-B. The bar represents the $\log _{2}$ fold change value.

As shown in Figure 2, we used TBtools [21] to draw a Venn diagram after comparison of DEPs between $(+) \mathrm{WT} /(-) \mathrm{WT}$ and $(+)$ sto/(+)WT groups. There were 221 STO-dependent UV-B response proteins and $39 \mathrm{UV}-\mathrm{B}$ dependent proteins in WT.

To further investigate the biological function of the STO-dependent UV-B-induced proteins, we performed a GO (gene ontology) analysis. As shown in Figure 3, STO-dependent UV-B response proteins were distributed in the CC (cellular component) including cellular, membranes, organelles (chloroplasts, thylakoids, and mitochondria), mainly involved in UV-B light response stimulation, metabolism, plant development, and other important BP (biological processes), with the MF (molecular function) of activating transcription by binding promoters and combining with chlorophyll. Furthermore, we performed a GO enrichment analysis in CC. From the results of GO enrichment (Figure 4), it can be found that the UV-B response proteins dependent on STO were mainly located on the photosynthesis membrane, inner capsule membrane, chloroplast membrane, and thylakoid, indicating that STO is closely related to photosynthesis. 


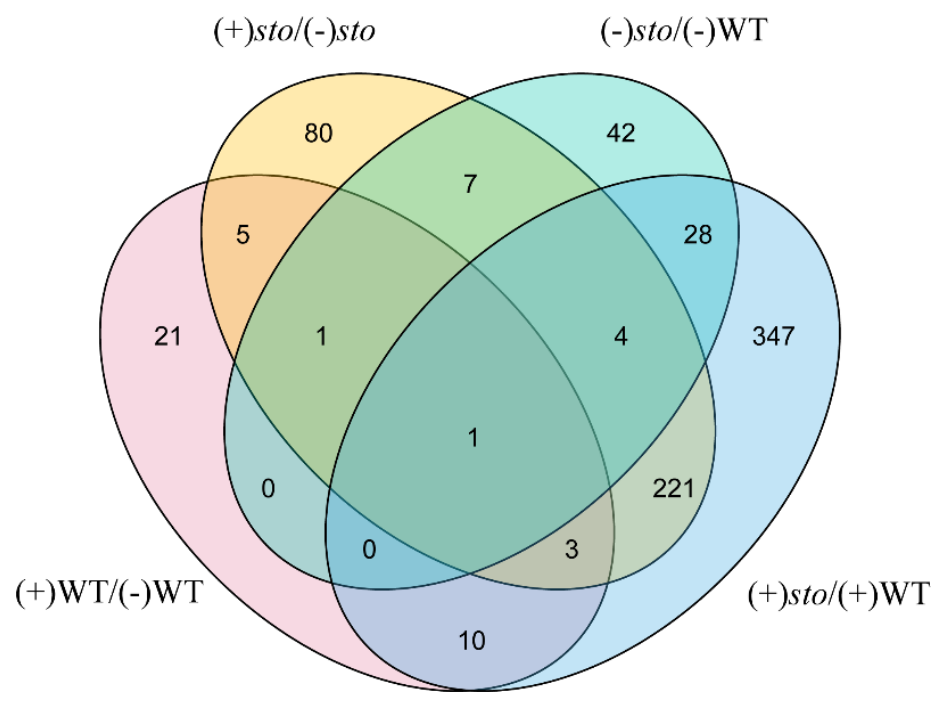

Figure 2. Venn diagram illustrating the number of identified proteins with abundance changed in WT and sto mutant before and after UV-B treatment.

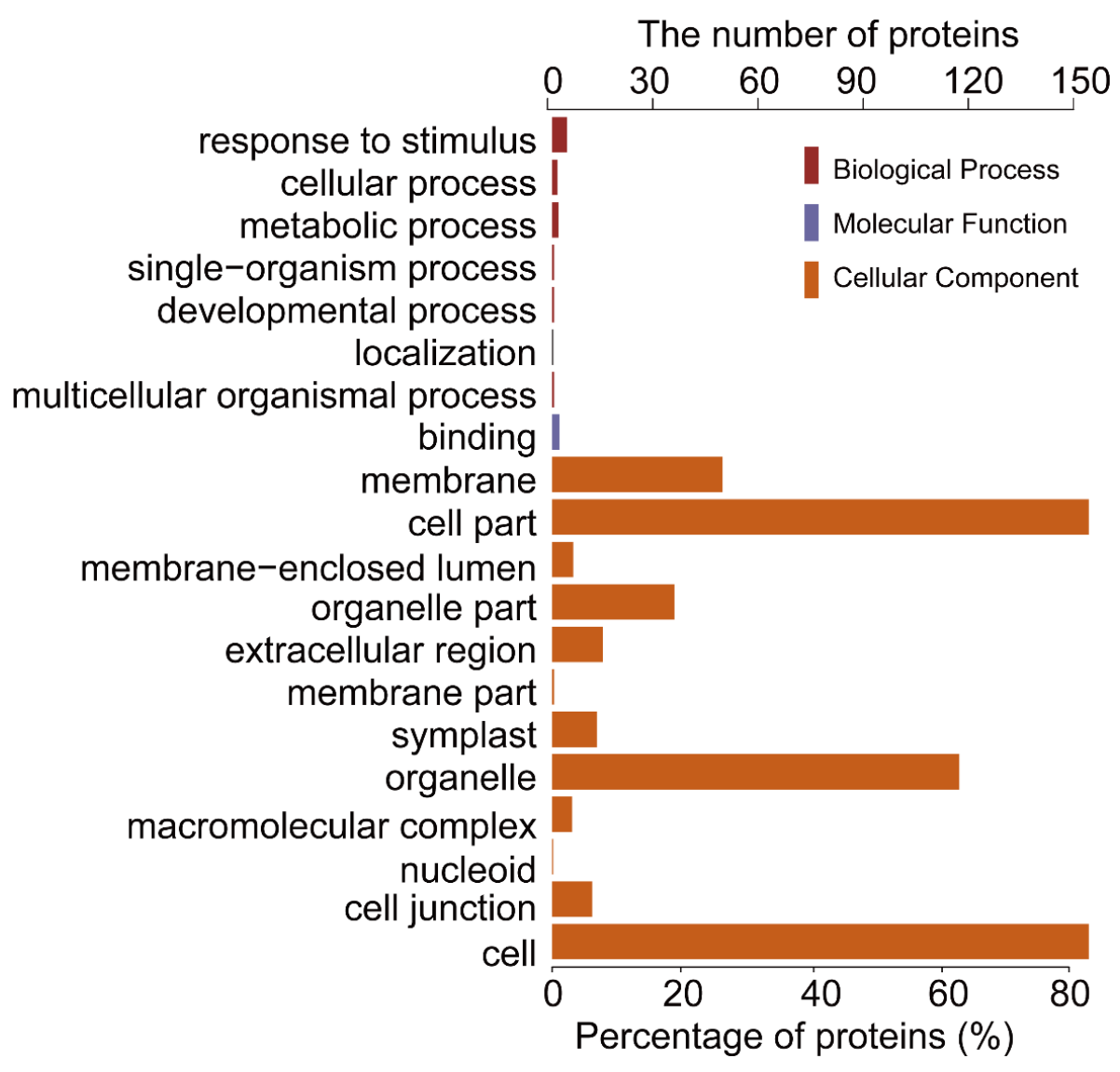

Figure 3. Gene ontology (GO) analysis of salt tolerance (STO)-dependent UV-B-induced proteins. GO analysis (gene ontology) describes the properties of genes and gene products in organisms from three aspects: the biological process (BP), the molecular function (MF), and the cell component (CC). 


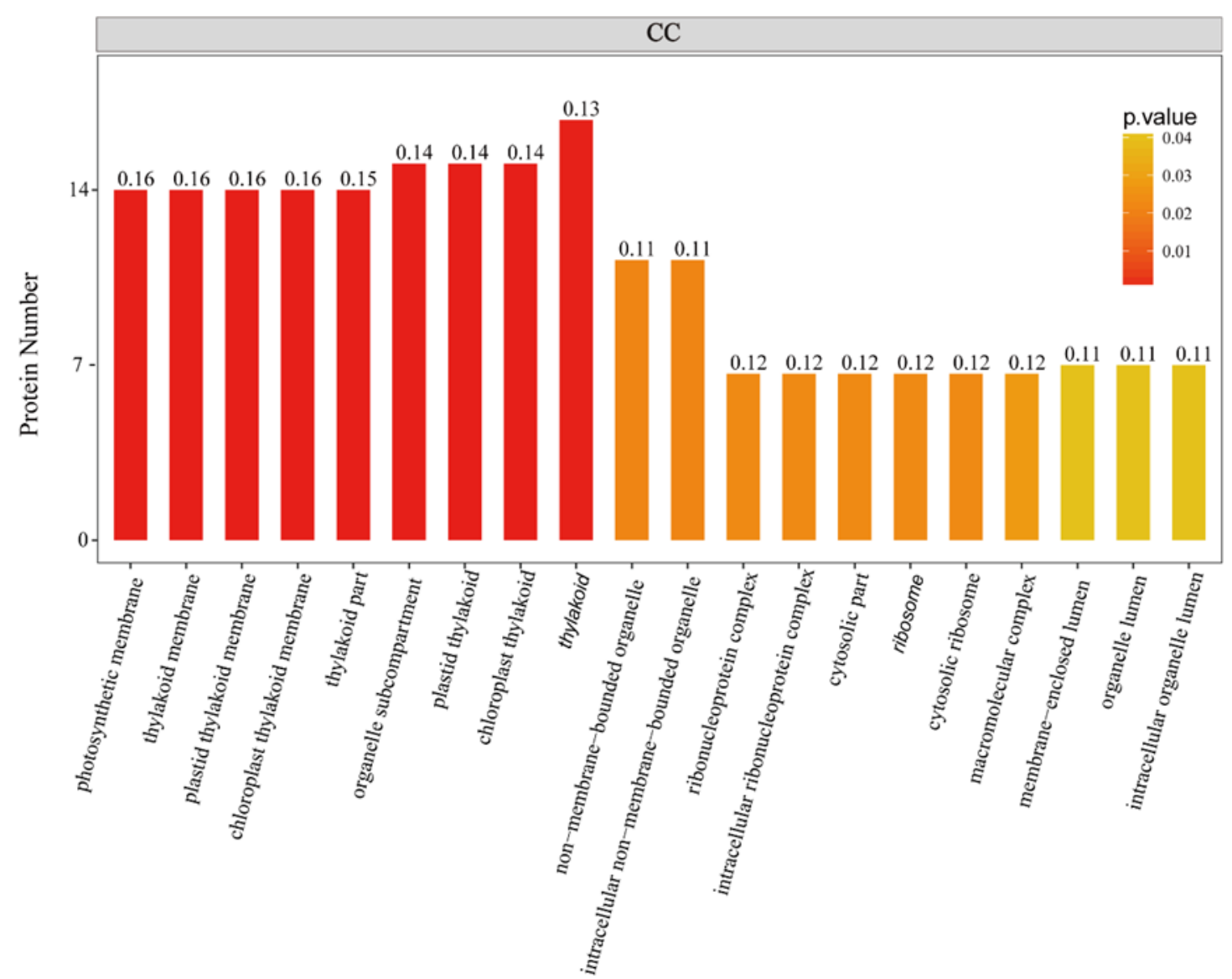

Figure 4. GO enrichment analysis of STO-dependent UV-B-induced proteins. The color gradient indicates the $P$ value. The smaller $P$ value indicates that the color is closer to red, the higher the level of GO enrichment. The number on the bar graph indicates the rich factor (rich factor $<=1$ ), referring to the percentage of differential proteins that are involved in this GO term.

It is difficult for a single protein to perform its biological functions. The proteins in the organism need to coordinate with each other in an orderly way to perform their specific biological functions effectively. Therefore, we enriched those differential proteins that performed the same biological functions by the KEGG (Kyoto Encyclopedia of Genes and Genomes) pathway. The results are as follows: $59 \%$ of the proteins in the antenna protein complex and $20 \%$ of the proteins in photosynthesis changed significantly (Figure 5), which affected the light-harvesting capacity, photoelectron transfer, and photophosphorylation of photosynthesis. Therefore, the STO-dependent UV-B response proteins are most related to the assembly of antenna protein complex and photosynthesis pathway.

In order to find the key regulatory factors, the STRING database and Cytoscape software were used to construct the protein interaction network (PPI) to analyze the hub proteins in the network. The STO-dependent UV-B response proteins were filtered by Cytoscape, the top 20 proteins with the highest connectivity were selected for the map, and finally 8 PPI interaction networks were obtained (Figure 6). The function of interacting proteins is as follws: AT5G65220, as a hub protein, interacting with many proteins participates in cellular process; VPS2.1 (vacuolar protein sorting-associated protein 2 homolog 1) interacting with VPS37-1 participates in protein transport; SAR3 (suppressor of Auxin resistance 3) interacting with AT1G10390 participates in transport; AT1G76200 interacting with AT1G49140 participates in the oxidation-reduction process; GPX1 (glutathione peroxidase 1) interacting with GSTF3 (glutathione S-transferase F3) participates in the metabolic process; PBB2 (proteasome beta subunit PBB2) interacting with MEE34 (maternal effect embryo arrest 34) participates in the metabolic process; and PGR5 (proton gradient regulation 5) directly interacting with OHP 
(encodes a one-helix protein homologous to cyanobacterial high-light inducible proteins) and indirectly interacting with LHCA2 (Photosystem I light-harvesting complex gene 2) and PSAD-2 (Photosystem I reaction center subunit II-2) participates in photosynthesis and light reaction.

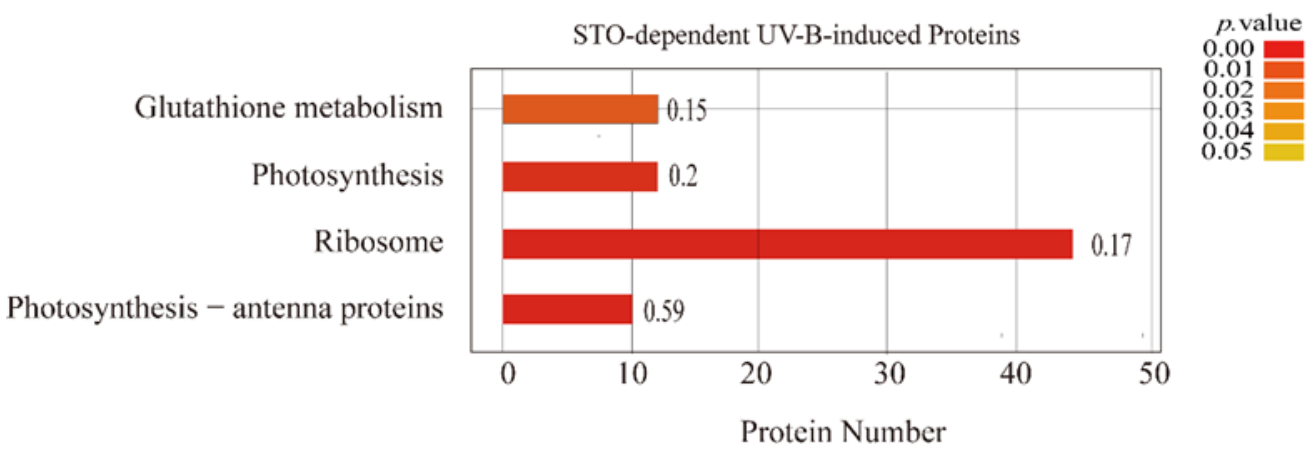

Figure 5. KEGG enrichment analysis of STO-dependent UV-B-induced proteins. The color gradient indicates the $P$ value. The smaller $P$ value indicates that the color is closer to red, the higher the level of pathway enrichment. The number on the bar graph indicates the rich factor (rich factor $<=1$ ), referring to the percentage of differential proteins that are involved in this pathway.

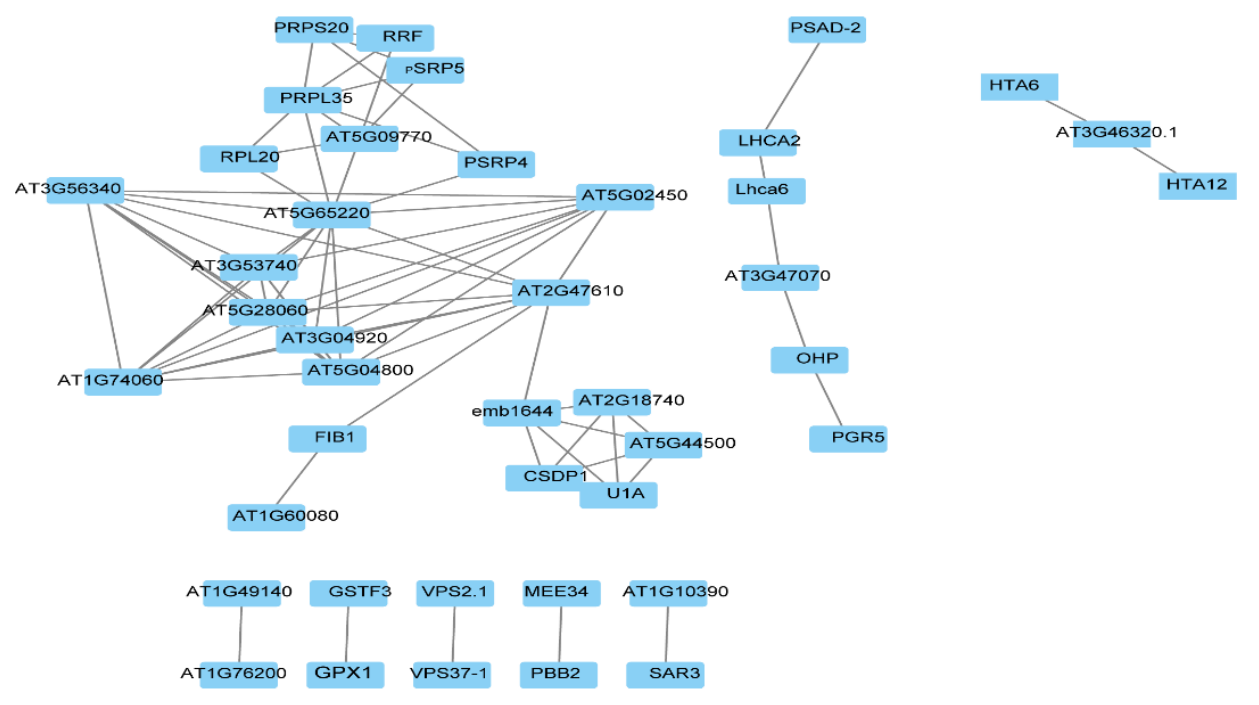

Figure 6. Protein interaction network (PPI) analysis of STO-dependent UV-B-induced proteins.

\subsection{Changes of Candidate Proteins in Each Treatment Group}

According to the results of enrichment analysis of GO, KEGG, and PPI protein interaction analysis, the proteins involved in antenna proteins and photosynthesis were listed. At the same time, considering the possible technical problems, the high-abundance proteins of plants were not removed in advance, and some low-abundance proteins (such as transcription factors) were not identified, leading to the failure of enrichment of some pathways. The proteins of flavonoid synthesis and metabolism and hormone signal transduction pathways were also listed for analysis. UV-B radiation promotes the accumulation of flavonoids and anthocyanins, changes hormone synthesis and signal regulation, and activates the anti-stress mechanism in plants. NS in the table indicates that the difference is not significant $(P<0.05)$, and the ratio is the multiple of protein expression difference (ratio $>1.2$, indicating up-regulated; ratio $<0.83$, indicating down-regulated). After summarizing the changes of different proteins in different treatment groups, it is speculated that the STO protein not only responds to UV-B, but also is closely related to stress response. The specific protein change information is as follows. 
(1) Changes of differential protein expression related to flavonoids synthesis and metabolism. The expression of differential proteins from (+)WT/(-) WT and (+)sto/(-)sto including phenylalanine lyase1/2 (PAL1/2), chalcone synthetase (CHS), flavonoid synthetase (FLS), and UDP glycosyltransferase were higher than that without UV-B radiation. However, the key enzymes of the flavonoid pathway in sto mutants were also significantly up-regulated after UV-B radiation. Compared with the WT, the sto mutant after UV-B radiation showed no significant difference. The results show that UV-B notably induces and accumulates flavonoids in plants to adapt to the environment. The accumulation of flavonoids do not depend on STO, and there are other proteins to regulate the expression of flavonoids (Table 3).

Table 3. Fold changes of flavonoid pathway proteins in different groups.

\begin{tabular}{cccccc}
\hline Gene ID & Description & $\begin{array}{c}\mathbf{( + ) W T /} \\
(-) W T\end{array}$ & $\begin{array}{c}(+\mathbf{+}) \text { sto/ } \\
(-) \text { sto }\end{array}$ & $\begin{array}{c}\mathbf{( - )} \text { sto/ } \\
(-) \mathbf{W T}\end{array}$ & $\begin{array}{c}\mathbf{( + )} \text { sto/ } \\
\mathbf{( + ) W T}\end{array}$ \\
\hline AT2G37040.1 & PAL1, phenylalanine ammonia-lyase 1 & 1.6 & 1.31 & $1.13(\mathrm{NS})$ & 0.92 \\
AT3G53260.1 & PAL2, phenylalanine ammonia-lyase 2 & 1.35 & 1.18 & $0.99(\mathrm{NS})$ & 0.87 \\
AT5G13930.1 & CHS, chalcone and stilbene synthase family protein & 1.86 & 1.39 & 1.23 & 0.92 \\
AT5G08640.1 & Flavonol synthase 1 & 1.32 & 1.12 & $1.07(\mathrm{NS})$ & 0.9 \\
AT3G21560.1 & UDP-Glycosyltransferase 84A2 & 1.57 & 1.35 & $1.04(\mathrm{NS})$ & 0.89 \\
AT1G43620.1 & UDP-Glycosyltransferase superfamily protein & 1.22 & $1.05(\mathrm{NS})$ & $0.96(\mathrm{NS})$ & 0.82 \\
\hline
\end{tabular}

Note: Fold change more than 1.2 times is up-regulated, and less than 0.83 times is down-regulated, $P<0.05$.

'NS' means no significance.

(2) Changes of differential protein expression involved in photosynthesis. As shown in Table 4, there was no significant change in the expression of photosynthesis-related proteins in the WT before and after UV-B radiation. Similarly, there was no significant change in the expression of photosynthesis-related proteins in sto mutant and WT control groups without UV-B radiation. In the comparison group of $(+)$ sto/(-)sto and $(+)$ sto/(+)WT, the protein expressions of PSBs and LHCs decreased significantly, which indicates that the absence of STO under UV-B radiation would affect photosynthesis.

Table 4. Fold changes of differential proteins in the photosynthesis pathway in different groups.

\begin{tabular}{|c|c|c|c|c|c|}
\hline Gene ID & Description & $\begin{array}{l}(+) W T / \\
(-) W T\end{array}$ & $\begin{array}{l}(+) \text { sto/ } \\
(-) \text { sto }\end{array}$ & $\begin{array}{l}(-) \text { sto/ } \\
(-) W T\end{array}$ & $\begin{array}{l}(+) \text { sto/ } \\
(+) W T\end{array}$ \\
\hline AT2G05620.1 & $\begin{array}{c}\text { PGR5, response to water deprivation, photosynthetic } \\
\text { electron transport in PSI }\end{array}$ & 1.10(NS) & $0.90(\mathrm{NS})$ & 0.95(NS) & 0.78 \\
\hline AT3G54890.4 & $\begin{array}{l}\text { Chlorophyll binding component of the light } \\
\text { harvesting complex associated with PSI }\end{array}$ & $1.08(\mathrm{NS})$ & 0.93(NS) & $0.90(\mathrm{NS})$ & 0.77 \\
\hline AT1G31330.1 & PSAF, PSI subunit F & $1.11(\mathrm{NS})$ & $1.00(\mathrm{NS})$ & $0.91(\mathrm{NS})$ & 0.81 \\
\hline ATCG01010.1 & NADH dehydrogenase activity & 1.12(NS) & $0.92(\mathrm{NS})$ & 0.95 (NS) & 0.78 \\
\hline AT5G58260.1 & $\begin{array}{l}\text { Oxidoreductases, acting on NADH or NADPH, } \\
\text { quinone or similar compound as acceptor }\end{array}$ & $1.07(\mathrm{NS})$ & $0.96(\mathrm{NS})$ & $0.92(\mathrm{NS})$ & 0.82 \\
\hline AT3G01440.1 & Oxygen evolving enhancer 3 (PsbQ) family protein & 1.05 (NS) & 0.97(NS) & 0.9 & 0.83 \\
\hline ATCG00710.1 & $\begin{array}{c}\text { A component of the photosystem II } \\
\text { oxygen evolving core }\end{array}$ & 1.19 & $0.94(\mathrm{NS})$ & 1.04(NS) & 0.82 \\
\hline AT2G40100.1 & $\begin{array}{l}\text { LHCB4.3 (light harvesting complex PSc), } \\
\text { chlorophyll binding }\end{array}$ & 1.03(NS) & 0.85 & 0.97(NS) & 0.8 \\
\hline AT1G15820.1 & LHCB6, PSII antenna complex & 1.09 (NS) & $0.96(\mathrm{NS})$ & $0.92(\mathrm{NS})$ & 0.81 \\
\hline AT1G61520.2 & LHCA3, chlorophyll binding & $1.13(\mathrm{NS})$ & $0.93(\mathrm{NS})$ & $1.00(\mathrm{NS})$ & 0.83 \\
\hline AT2G05070.1 & $\begin{array}{l}\text { LHCB2.2, constitute the antenna system of the } \\
\text { photosynthetic apparatus, chlorophyll binding }\end{array}$ & $1.08(\mathrm{NS})$ & $0.98(\mathrm{NS})$ & $0.91(\mathrm{NS})$ & 0.83 \\
\hline AT1G19150.1 & $\begin{array}{l}\text { LHCA6, chlorophyll binding PSI type II } \\
\text { chlorophyll a/b-binding }\end{array}$ & $1.11(\mathrm{NS})$ & 0.94(NS) & 0.89 (NS) & 0.76 \\
\hline AT2G34430.1 & $\begin{array}{l}\text { LHB1B1, chlorophyll binding PSII type I } \\
\text { chlorophyll a/b-binding }\end{array}$ & 1.07(NS) & $0.91(\mathrm{NS})$ & 0.79 & 0.67 \\
\hline AT2G34420.1 & LHB1B2, chlorophyll binding & $0.94(\mathrm{NS})$ & 0.88 & 0.89 & 0.83 \\
\hline AT2G20890.1 & PSB29, controls the assembly of the PSII complex & 1.14(NS) & 0.99 (NS) & $0.91(\mathrm{NS})$ & 0.79 \\
\hline
\end{tabular}

Note: Fold change more than 1.2 times is up-regulated, and less than 0.83 times is down-regulated, $P<0.05$. 'NS' means no significance. 
(3) Changes of differential protein expression involved in hormones. In Table 5, the negative regulatory transcription factor ERD15 (early response to dehydration 15) of ABA (abscisic acid) signal significantly decreased 0.78 times in (+)WT/(-)WT, 1.6 times in (+)sto/(-)sto, and 1.9 times in $(+) s t o /(+) W T$, indicating that ERD15 may respond to UV-B radiation. STO may negatively regulate the expression of ERD15 in UV-B signal transduction. The proteins P5CS1 (delta-1-pyrroline-5-carboxylate synthase 1), RD22 (BURP domain protein RD22), and LTP3 (lipid-transfer protein 3) related to ABA signal transduction were significantly down-regulated in $(+)$ sto/ $(+) \mathrm{WT}$, indicating that STO may promote the expression of proteins related to ABA signal transduction, but not relate to UV-B radiation. Lox2 (lipoxygenase 2) related to JA (jasmonate) was significantly down-regulated in (-)sto/(-)WT and $(+) s t o /(+) \mathrm{WT}$, indicating that STO promotes Lox2 protein expression and communicated with JA signal, but does not depend on UV-B radiation. DRM2 (DNA (cytosine-5)-methyltransferase) related to IAA (auxin) and methylation was down-regulated in (+)WT/(-)WT and up-regulated in $(+)$ sto/(+)WT, suggesting that STO may inhibit the expression of DRM2 in UV-B signal transduction and reduce the degree of methylation in plants. Auxin efflux carrier family protein and AXR4 (auxin response 4) were significantly up-regulated in (+)sto/(-)sto and $(+)$ sto/(+)WT, indicating that STO may inhibit the expression of auxin efflux carrier family protein and AXR4 under UV-B radiation, and communicate with IAA signals. ACO2 (aconitate hydratase 2)-related ethylene was significantly up-regulated in $(+)$ sto/(-)sto and (+)sto/(+)WT, indicating that STO inhibits the expression of $\mathrm{ACO} 2$, but does not depend on UV-B radiation.

Table 5. Fold changes of hormone-related proteins.

\begin{tabular}{|c|c|c|c|c|c|}
\hline Gene ID & Description & $\begin{array}{l}(+) W T / \\
(-) W T\end{array}$ & $\begin{array}{l}(+) \text { sto/ } \\
(-) \text { sto }\end{array}$ & $\begin{array}{l}(-) s t o / \\
(-) W T\end{array}$ & $\begin{array}{l}(+) \text { sto/ } \\
(+) W T\end{array}$ \\
\hline AT2G41430.1 & $\begin{array}{c}\text { ERD15, an attenuator of plant } \mathrm{ABA} \text {, response to light } \\
\text { intensity, water deprivation, cold tolerance, } \\
\text { salt stress }\end{array}$ & 0.78 & 1.62 & 0.92(NS) & 1.91 \\
\hline AT2G39800.1 & $\begin{array}{c}\text { P5CS1, response to ABA, desiccation, oxidative } \\
\text { stress, salt stress, water deprivation, } \\
\text { root development }\end{array}$ & 1.00(NS) & 1.09(NS) & 0.75 & 0.82 \\
\hline AT5G25610.1 & $\begin{array}{c}\text { RD22, response to abscisic acid, response to } \\
\text { desiccation, salt stress }\end{array}$ & 0.97(NS) & $0.91(\mathrm{NS})$ & 0.8 & 0.75 \\
\hline AT5G59320.1 & $\begin{array}{c}\text { LTP3, involved in lipid transport, response to ABA, } \\
\text { water deprivation }\end{array}$ & 1.19(NS) & 0.99 (NS) & $0.78(\mathrm{NS})$ & 0.69 \\
\hline AT5G01990.1 & Auxin efflux carrier family protein & $0.94(\mathrm{NS})$ & 1.79 & $0.86(\mathrm{NS})$ & 1.64 \\
\hline AT1G54990.1 & AXR4/RGR1, auxin-resistant root growth & 1.04(NS) & 1.4 & 1.04 & 1.41 \\
\hline AT2G33830.1 & DRM2, response to stress and environmental factors & 0.76 & 0.86 & 1.11 & 1.26 \\
\hline AT3G45140.1 & $\begin{array}{l}\text { LOX2, response to water deprivation, JA, } \\
\text { oxidation-reduction process, lipid oxidation }\end{array}$ & 1.03(NS) & 1.02(NS) & 0.79 & 0.78 \\
\hline AT1G62380.1 & $\begin{array}{l}\text { ACO2, response to fatty acid, defense response, } \\
\text { ethylene stimulus, ethylene biosynthetic process, } \\
\text { oxidation-reduction process, cytokinin, salt stress }\end{array}$ & $0.97(\mathrm{NS})$ & 0.93(NS) & 1.4 & 1.34 \\
\hline
\end{tabular}

Note: Fold change more than 1.2 times is up-regulated, and less than 0.83 times is down-regulated, $P<0.05$.

'NS' means no significance.

\subsection{Verification of Differential Protein}

(1) qRT-PCR verification of differential genes related to flavonoid synthesis and metabolism. The results of proteomics show that UV-B significantly induces the accumulation of flavonoids in plants, and the accumulation of flavonoids do not depend on STO (Table 3). Therefore, the transcriptional levels of PAL1, CHS, and FLS in the flavonoid biosynthesis pathways of WT, sto mutant, and 35S-STO-GFP plants were detected after UV-B radiation for $4 \mathrm{~h}$. As shown in Figure 7, PAL1 in WT, sto mutant, and 35S-STO-GFP plants were up-regulated by more than 5 times after UV-B radiation, but the expression levels of PAL1 in sto mutant and 35S-STO-GFP plants were significantly lower than that in $\mathrm{WT}$, indicating that STO does not regulate the transcription level of PAL1 in UV-B signaling. The CHS in WT, sto mutant, and STO overexpressing plants increased by more than 40 times after UV-B radiation, but the expression level of $\mathrm{CHS}$ in sto mutant was significantly lower than that in WT and STO 
overexpressing plants, indicating that STO may regulate the transcription level of $\mathrm{CHS}$ in the UV-B pathway. FLS in WT, sto mutant, and 35S-STO-GFP plants were up-regulated by more than 100 times after UV-B radiation, but FLS expression level in 35S-STO-GFP plants was significantly lower than that in WT and sto mutant, and there was no significant difference in FLS transcription levels between WT and sto mutant. It is suggested that the up-regulated expression of FLS after UV-B radiation does not depend on STO, but STO overexpression can promote FLS transcription and flavonoid accumulation.

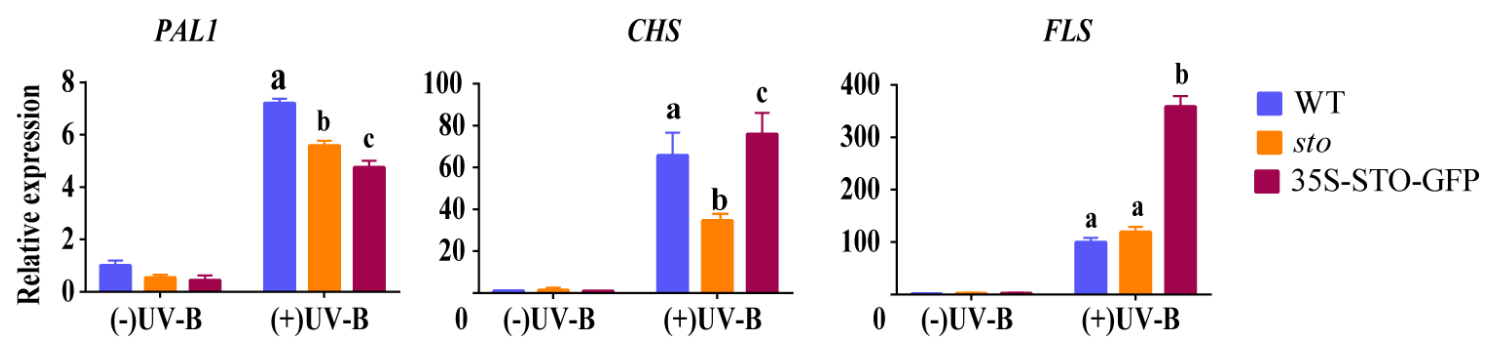

Figure 7. Expression profile of genes involved in flavonoid synthesis before and after UV-B treatment. The WT, sto, and 35S-STO-GFP seedlings were grown in white light for seven days and then subjected to a four-hour UV-B treatment $\left(0.6 \mathrm{~W} / \mathrm{m}^{2}\right)$. Error bars indicate the SEM of three independent biological and technical replicates. Different letters indicate significant differences between gene relative expressions of those seedlings grown under UV-B radiation $(P<0.05$, Dunnett's multiple). Actin2 was used as an internal reference gene.

(2) qRT-PCR verification of differential genes involved in photosynthesis. The results of proteomics show that STO relates to the antenna protein (Table 4). The transcription levels of PSAD-2, LHCA2, and LHCA3 (Photosystem I light harvesting complex gene 3) in WT, sto mutant, and 35S-STO-GFP plants were measured after $4 \mathrm{~h}$ UV-B radiation. As shown in Figure 8, the gene expression levels of PSAD-2, LHCA2, and LHCA3 in 35S-STO-GFP lines were significantly higher than those in WT and sto mutant without UV-B radiation, indicating that STO can improve the photosynthesis of plants. After UV-B radiation, $P S A D-2, L H C A 2$, and $L H C A 3$ were significantly down regulated in WT, sto mutants, and 35S-STO-GFP plants after UV-B radiation, indicating that UV-B radiation affects photosynthesis of plants. After UV-B radiation, the PSAD-2 gene expression level of STO overexpressed plants was higher than that of WT, the LHCA2 gene expression level was higher than that of WT and sto mutant, and the LHCA3 gene expression level was higher than that of sto mutant. The results indicate that STO could restore the photosynthetic capacity after UV-B radiation and reduce the light damage caused by UV-B.
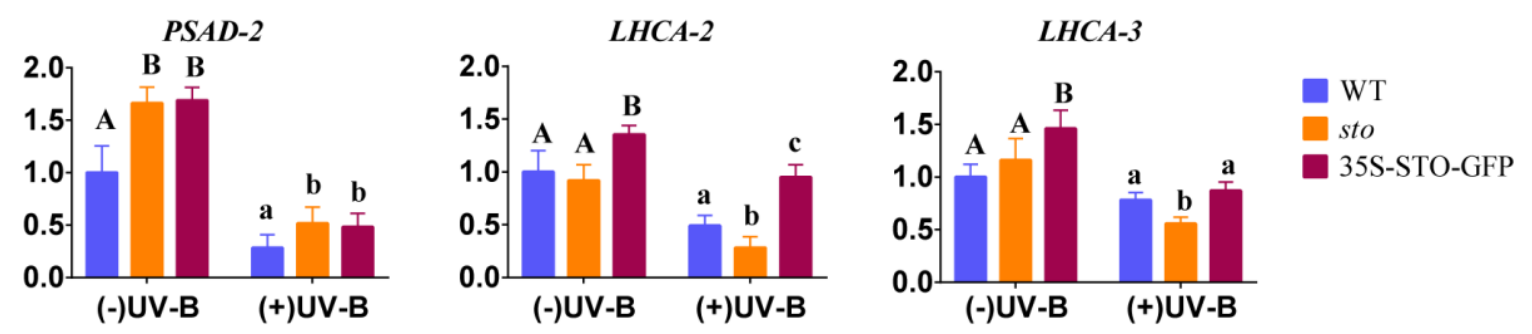

Figure 8. Expression profile of photosynthesis genes before and after UV-B treatment. The WT, sto, and 35S-STO-GFP seedlings were grown in white light for seven days and then subjected to a four-hour UV-B treatment $\left(0.6 \mathrm{~W} / \mathrm{m}^{2}\right)$. Error bars indicate the SEM of three independent biological and technical replicates. Different capital letters and lowercase letters indicate significant differences between gene relative expression of those seedlings grown without or with UV-B radiation, respectively $(P<0.05$, Dunnett's multiple). Actin2 was used as an internal reference gene.

(3) qRT-PCR verification of differential genes involved in hormones. To gain further insight into the crosstalk between UV-B and the ABA pathway, we measured the transcript levels of RD22, AB15 
(ABA insensitive 5), P5CS1, SAD2, DREB1A (dehydration response element B1A, encodes a member of the DREB subfamily A-1 of ERF/AP2 transcription factor family (CBF3)), and DREB2A (dehydration response element $\mathrm{B} 2 \mathrm{~A}$ ) under $\mathrm{UV}-\mathrm{B}$ radiation (Figure 9). RD22, AB15, and P5CS1 were down-regulated in the WT and 35S-STO-GFP seedlings but were up-regulated in sto mutant, indicating the inhibition of $R D 22, A B 15$, and P5CS1 transcription by STO under UV-B radiation. The transcript levels of SAD2 do not have a significant difference between all the genotypes, indicating that UV-B does not affect $S A D 2$ transcription. DREB1A and DREB2A were significantly up-regulated after UV-B treatment, indicating that these two genes activate by STO in a UV-B-dependent manner (Figure 9). These results provide evidence for a crosstalk between STO and ABA signaling.
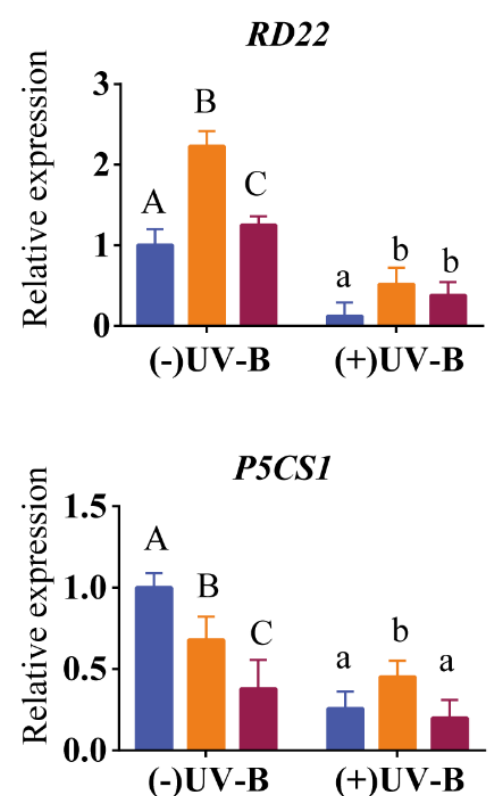

$A B I 5$

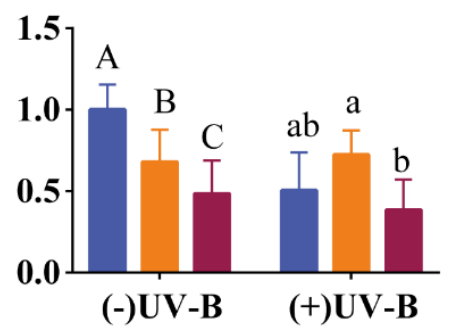

DREB1A

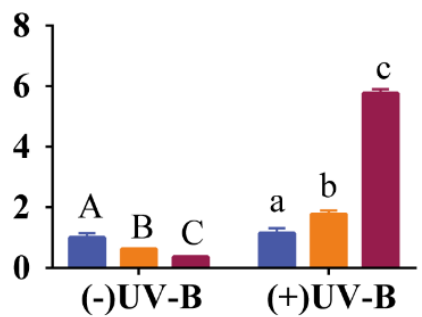

$S A D 2$

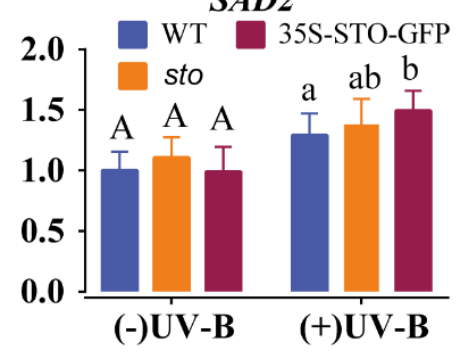

Figure 9. Expression profile of ABA pathway genes before and after UV-B treatment. The WT, sto, and 35S-STO-GFP seedlings were grown in white light for seven days and then subjected to a four-hour UV-B treatment $\left(0.6 \mathrm{~W} / \mathrm{m}^{2}\right)$. Error bars indicate the SEM of three independent biological and technical replicates. Different capital letters and lowercase letters indicate significant differences between gene relative expression of those seedlings grown without or with UV-B radiation, respectively $(P<0.05$, Dunnett's multiple). Actin2 was used as an internal reference gene.

(4) PRM (parallel reaction monitoring) verification of differential proteins. To validate the iTRAQ data, we selected five proteins within the potentially relevant pathways (anthocyanin biosynthesis, photosynthesis, and protein synthesis) for PRM quantification (Table 6). The PRM results demonstrated the consistency in the differential expression patterns of selected proteins with the iTRAQ data. LHCB6, PSAD-2, and RPL5B were up-regulated in $(+) \mathrm{WT} /(-) \mathrm{WT}$ and down-regulated in $(+)$ sto/(+)WT. In $(+) W T /(-) W T$, the expression levels of PAL1 and UGT84A2, which are involved in anthocyanin biosynthesis, show a significant increase in both the PRM and iTRAQ analyses. In (+)sto/(+)WT, PAL1 and UGT84A2 were up-regulated in the PRM data and were unchanged according to the iTRAQ results. In general, consistency between the PRM and the iTRAQ results validated the credibility of ITRAQ in proteomic analyses. 
Table 6. Representative protein quantitative confirmation with PRM analysis.

\begin{tabular}{|c|c|c|c|c|c|c|c|c|c|c|}
\hline \multirow{2}{*}{$\begin{array}{l}\text { Accession } \\
\text { Number }\end{array}$} & \multirow{2}{*}{$\begin{array}{c}\text { Gene } \\
\text { Symbol }\end{array}$} & \multirow{2}{*}{ Signature Peptides } & \multicolumn{4}{|c|}{ PRM } & \multicolumn{4}{|c|}{ iTRAQ } \\
\hline & & & $\mathbf{A}$ & B & $\mathrm{C}$ & D & $\mathbf{A}$ & B & $\mathrm{C}$ & D \\
\hline AT2G37040.1 & PAL1 & $\begin{array}{l}\text { LAGISSGFFDLQPK } \\
\text { FLNAGIFGSTK } \\
\text { EELGTELLTGEK }\end{array}$ & 2.89 & 1.95 & 1.60 & 1.14 & 1.60 & 1.3 & 1.13 & 0.92 \\
\hline AT3G21560.1 & UGT84A2 & $\begin{array}{l}\text { YDFFDDGLPEDDEASR } \\
\text { IVEWCSQEK }\end{array}$ & 2.59 & 2.59 & 1.14 & 1.30 & 1.57 & 1.35 & 1.04 & 0.89 \\
\hline AT1G15820.1 & LHCB6 & $\begin{array}{c}\text { WVDFFNPDSQSVEWATPWSK } \\
\text { DGVYEPDFEK } \\
\text { SWIPAVK }\end{array}$ & 1.16 & 1.07 & 1.00 & 0.79 & 1.09 & 0.96 & 0.92 & 0.81 \\
\hline AT1G03130.1 & PSAD-2 & $\begin{array}{c}\text { AQVEEFYVITWNSPK } \\
\text { ITYQFYR } \\
\text { EGPNLLK }\end{array}$ & 1.31 & 0.66 & 1.14 & 0.52 & 1.10 & 0.94 & 0.91 & 0.78 \\
\hline
\end{tabular}

Note: The letter A means (+)WT/(-)WT, B means (+)sto/(-)sto, C means (-)sto/(-)WT, D means (+)sto/(+)WT.

\subsection{STO Regulates Hypocotyl Elongation and Protects the Photosynthetic System after UV-B Treatment}

In the absence of UV-B radiation the hypocotyls of the sto mutant were shorter than those of the WT and 35S-STO-GFP seedlings. However, the inhibitory effect of UV-B radiation on hypocotyl growth was compromised in 35S-STO-GFP. The sto mutants were more sensitive to UV-B (the hypocotyl length ratio of + UVB/-UV-B was 0.45 for the sto mutant, 0.54 for the WT, and 0.64 for 35S-STO-GFP). The hypocotyls of the 35S-STO-GFP seedlings were longer than those of the WT and sto mutant seedlings after UV-B treatment (Figure 10A-C). The values of Fv/Fm in WT, 35S-STO-GFP, and the sto mutant showed no obvious changes and ranged from 077 to 0.79 within $24 \mathrm{~h}$ after UV-B radiation, but NPQ in the 35S-STO-GFP seedlings was much higher than that of WT and the sto mutant. NPQ peaked at $12 \mathrm{~h}$ after UV-B radiation in all three genotypes (Figure 10D,E). After the UV-B treatment, the anthocyanin (UV-absorbing compounds) content in the sto mutant increased by 4 -fold, while the level of flavonoid remained the same as the sto mutant. A 2-fold increase in flavonoid level was observed in 35S-STO-GFP, and the anthocyanin level was doubled upon UV-B radiation. The level of anthocyanin increased by 2 -fold in the WT, whereas flavonoid content was only slightly elevated under UV-B treatment. According to the results above, UV-B exposure accelerated anthocyanin accumulation in the sto mutant (Figure 10F,G). Taken together, these phenotypes demonstrate that STO positively regulates hypocotyl elongation by antagonizing UV-B repression. These results also indicate flavonoids and STO play a significant role in plant photoprotection upon UV-B exposure.

A

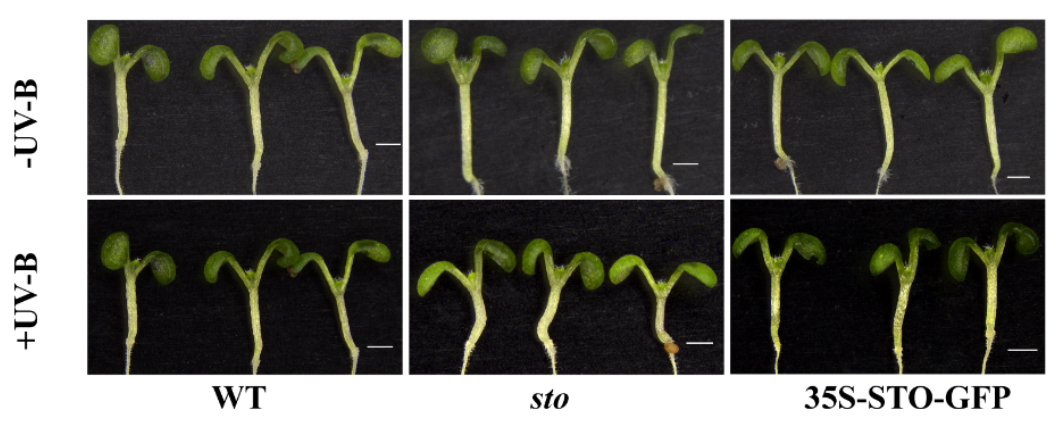

Figure 10. Cont. 

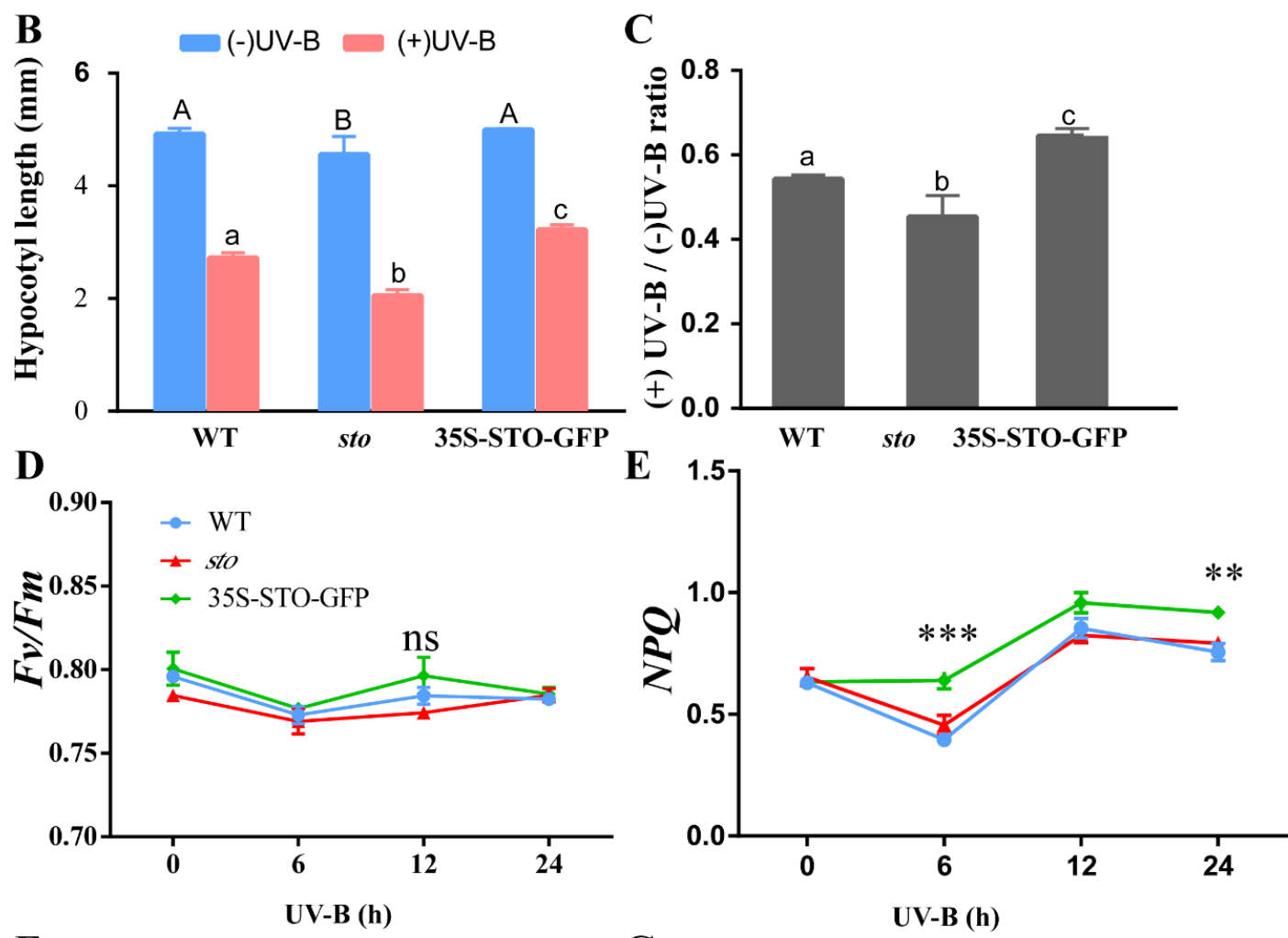

F

G

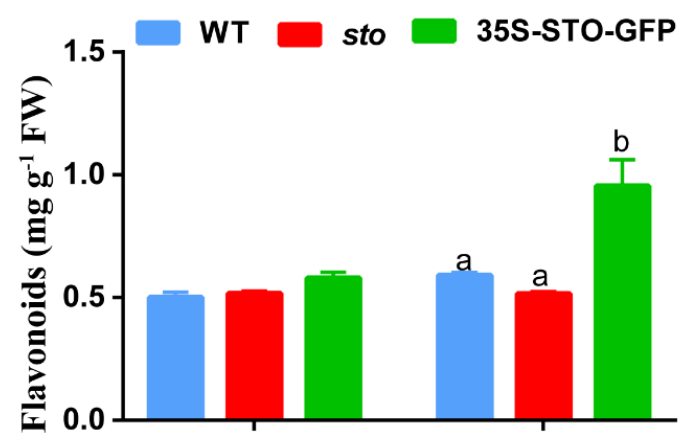

(-) UV-B

(+)UV-B

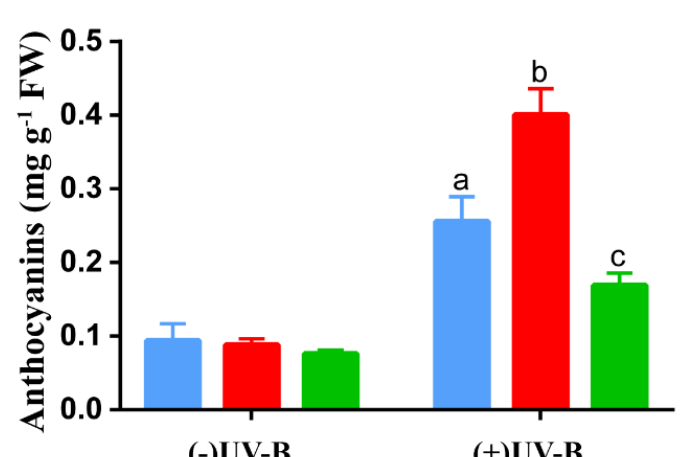

Figure 10. Physiological responses of STO under UV-B radiation. (A) Images of the six-day-old sto, $\mathrm{WT}$, and 35S-STO-GFP seedlings with or without UV-B radiation. UV-B radiation was $0.6 \mathrm{~W} / \mathrm{m}^{2}$; scale bar $=1 \mathrm{~mm}$. (B) Hypocotyl length of the sto, WT, and 35S-STO-GFP seedlings with or without UV-B radiation. Data are expressed as means \pm standard deviation (SD); $n>30$. The upper- and lower-case letters indicate the results of the Dunnett's multiple comparisons test on the difference in hypocotyl length between those seedlings without or with UV-B exposure, respectively $(P<0.05)$. (C) The grey bars show the ratio of hypocotyl length with or without UV-B radiation (+UV-B/-UV-B). Data are shown as means \pm SD $(n>30)$. Different letters indicate statistically significant differences $(P<0.05$, one-way ANOVA). (D) and (E) Fv/Fm and NPQ of sto, WT, and 35S-STO-GFP seedlings after UV-B exposure. The seedlings were grown in white light for seven days and then exposed to UV-B $\left(0.6 \mathrm{~W} / \mathrm{m}^{2}\right)$. Fv/Fm and NPQ were measured using CF Imager (Technologica). Data are shown as mean \pm SD $(n>20)$. "** indicates a statistically significant difference, and "ns" means no significant difference ( $P<0.05$, Dunnett's multiple comparisons test). (F) and (G) Anthocyanin and flavonoid contents in the seedlings with or without UV-B radiation. The seedlings were grown in the soil under white light for $12 \mathrm{~d}$ and then subjected to a three-day UV-B $\left(0.6 \mathrm{~W} / \mathrm{m}^{2}\right)$ exposure. The seedlings were then measured for anthocyanin and flavonoid contents. Data are shown as means \pm SEM $(n=6)$. Different letters indicate significant difference $(P<0.05$, Dunnett's multiple comparisons test). 


\subsection{STO Affects the Content of $A B A, J A$, and IAA upon UV-B Exposure}

The promoters of STO have response elements of IAA, ABA, and JA [22]. Our proteomics data indicate STO has a crosstalk with ABA, JA, and IAA signaling. Therefore, we measured the contents of these hormones in WT and the sto mutant upon UV-B exposure. LC-MS/MS analysis revealed an increase in ABA level in the WT and sto mutant after UV-B radiation. JA content in the WT slightly decreased after UV-B radiation. Interestingly, a sharp increase in JA was observed in the sto mutant $4 \mathrm{~h}$ after UV-B radiation, followed by a decrease to the normal level at $6 \mathrm{~h}$. IAA level in the WT and the sto mutant decreased after UV-B radiation (Figure 11). These results suggest that UV-B may inhibit hypocotyl elongation by inhibiting IAA synthesis or promoting ABA synthesis.

A

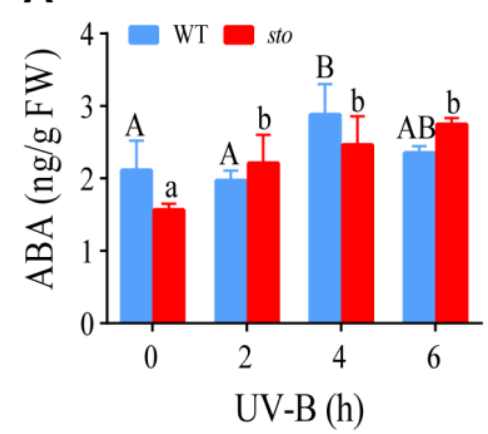

B

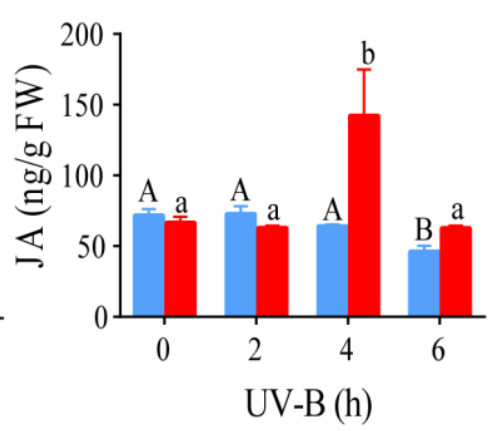

C

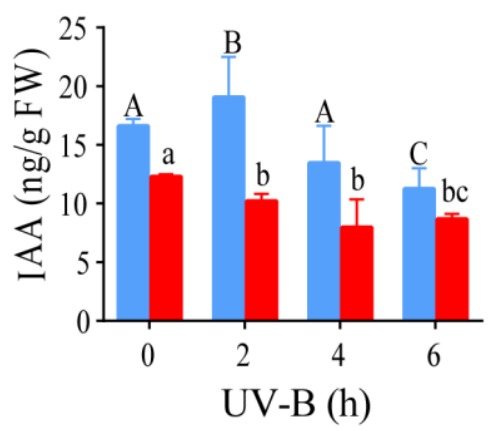

Figure 11. Changes of endogenous hormones ABA, JA, and IAA in wild-type and sto mutant under UV-B radiation. The seedlings of WT and sto were treated with UV-B $\left(0.6 \mathrm{~W} / \mathrm{m}^{2}\right)$ for 2,4 , and $6 \mathrm{~h}$, respectively. The seedlings in the soil grew under white light for $12 \mathrm{~d}$, then irradiated with UV-B $\left(0.6 \mathrm{~W} / \mathrm{m}^{2}\right)$. Error bars indicate the SEM of three independent biological and technical replicates. Different capital letters and lowercase letters indicate significant differences between changes of endogenous hormones of those seedlings grown without or with UV-B radiation, respectively $(P<0.05$, Dunnett's multiple).

\section{Discussion}

Many studies have reported the UV-B signaling pathway in Arabidopsis. UVR8 is a major photoreceptor [11], and the COP1-SPA (suppressor of PHYA-105) complex and many other key factors are also involved in UV-B photomorphogenesis, e.g., CUL4-DDB1 (cullin 4-DNA damage binding protein 1) [2,23], STO [15], BES1 and BIM1 [14], WRKY36 [13], HY5 [12], and RUP1 (repressor of UV-B photomorphogenesis 1) and RUP2 (repressor of UV-B photomorphogenesis 2) [24]. However, the orchestration of the downstream UV-B signaling pathway by STO is still poorly understood. Our study explores the regulatory network of STO using the iTRAQ method in Arabidopsis and demonstrates that STO could interfere with anthocyanin biosynthesis, promote the accumulation of flavonoid biosynthesis and antenna proteins, and crosstalk with the ABA pathway upon UV-B radiation.

\subsection{The Accumulation of Flavonoids and Anthocyanins under UV-B Radiation is Independent of STO}

As a small part of the solar spectrum, UV-B radiation has a significant impact on plant secondary metabolism, especially the accumulation of flavonoids (flavonoids and anthocyanins), which is considered to be one of the most important protective reactions to UV-B radiation. Flavonoids can filter UV-B before the UV-B signal is transmitted to sensitive molecules in mesophyll cells and causes oxidative degradation of membrane lipids [25-27]. However, UV-B damages DNA, proteins, and membranes, and it hinders photosynthesis and plant growth [28]. Our results show that UV-B increases the transcription (Figure 7) and protein levels (Table 3) of key enzymes in flavonoid synthesis, but their expressions do not depend on STO.

UV-B has a minor direct effect on photosynthesis but induces the accumulation of UV-absorbing flavonoids and anthocyanin under UV-B (Figure 10). In many plant species, UV-B up-regulates 
the expression of the phenylpropanoid and flavonoid biosynthetic genes [29]. Flavonoids and anthocyanin function as sunscreen substances [30]. Several biosynthetic enzymes, such as PAL, CHS, FLS, and UGT84A2, are up-regulated under UV-B light (Table 3). PAL initiates the synthesis of anthocyanin and other flavonoids, whereas CHS produces tetrahydroxy-chalcone [31]. FLS converts dihydroflavonol to flavonoids, whereas UFGT is an anthocyanin synthase that stabilizes anthocyanin [32]. Several flavonol and anthocyanin biosynthetic genes can be activated by HY5 and R2R3-MYBs [33]. However, a small amount of anthocyanin could still be detected in the tomato hy 5 mutant, indicating the existence of some HY5-independent transcription factors that mediate anthocyanin metabolism [34].

The flavonoid content was increased, and the anthocyanin content was decreased in the 35S-STO-GFP seedlings after UV-B induction (Figure 10). UGT84A2, a key anthocyanin biosynthetic enzyme, was up-regulated in the sto mutant (Table 6). The E3 ligase CUL4-DDB1-RUP1/RUP2 complex mediates HY5 degradation under UV-B radiation, thus inhibiting UV-B-induced anthocyanin accumulation. The transcript levels of CHS, UGT84A, ELIP1, and ELIP2 were up-regulated in double-mutant rup1-1 rup2-1 seedlings. Meanwhile, COP1 directly targets RUP1/RUP2 for degradation, which alleviates RUP1/RUP2 accumulation and COP1-HY5, thus stabilizing HY5 under UV-B radiation [35]. The accumulation of anthocyanin in three myb mutants was higher than that in hy5 mutants, indicating that the accumulation of anthocyanin must require the accumulation of HY5 [36]. In Arabidopsis, flavonoid metabolism is precisely controlled. For example, miR156-mediated transcript cleavage reduces anthocyanin accumulation and enhances flavonoid accumulation [37]. MYB, bHLH, and WD40 are the main transcription factors that regulate anthocyanin synthesis, whose protein complexes (MBW) are bound to the promoters of structural genes for regulation [38-40]. COP1 and SPA (suppressor of phya-105) inhibit photoinduced anthocyanin biosynthesis by targeting the key active factors (PAP1, PAP2, HY5, and their homologues, HYH) in anthocyanin synthesis in Arabidopsis [41]. mir828/TAS4-siR81(-) negatively regulates anthocyanin accumulation by inhibiting the expression of positive regulatory factors PAP1, PAP2, and MYB113 [42].

How STO regulates the balance between anthocyanin and flavonoids is still unknown. STO may affect proteins related to the synthesis of flavonoids by regulating the transcription of HY5. However, the expression of HY5 protein was not identified in the differential proteomic data, which may be due to the low expression of transcription factors covered by high-abundance proteins, resulting in many reported transcription factors not identified.

\subsection{STO Promotes Photosynthesis-Antenna Protein Accumulation}

Photosynthesis is the most important chemical reaction on the earth, which can provide energy and material sources for life activities. Photosystem II (PSII) and photosystem I (PSI) promote the absorption, transmission, and transformation of light energy in photosynthesis. In the face of adversity, plants reduce energy supply and resist various abiotic stresses by inhibiting photosynthetic and energy release reaction. Photosynthetic electron transport and many metabolic reactions are carried out in chloroplasts, and environmental stress easily affects the metabolism balance of chloroplasts. PSI-LHCI in green algae is combined with more antenna protein complexes, indicating that an increase in the ratio of pigment/reaction center of antenna protein complexes is conducive to the PSI capturing more photons [43]. With the enhancement of UV-B radiation, the ability of electron transfer in plants decreases, the content of Cytf decreases, PSI protein damages, and ATPase and photophosphorylation activity decreases [44-47].

To protect the photosynthetic machinery, UV-B significantly induces the quantum efficiency (qE) capacity in Chlamydomonas reinhardtii, as several key contributors to $\mathrm{qE}$, including LHC Stress-Related 1 (LHCSR1) and PSBS, are up-regulated by UVR8 [48]. NPQ also participates in the regulation of light harvesting, dissipating excess energy as heat through the major and most rapid component, $\mathrm{qE}$ (energy-dependent component), to circumvent photodamage [49]. PsbS belongs to the LHC protein superfamily [50] and is essential for $\mathrm{qE}$ [51]. In Arabidopsis, PSB29/THF1 is important for the normal accumulation of the FtsH heterocomplex involved in PSII repair [52]. The antenna system 
is composed of proteins of the LHC family and antenna complexes that protect photosystem I from photoinhibition [53]. NPQ also requires lipocalin proteins [54,55] such as V de-epoxidase (VDE) and the thylakoid membrane stromal-located zeaxanthin epoxidase (ZEP) [56]. Therefore, interference with NPQ may be caused by the down-regulation of lipid transporter proteins in the thylakoid.

Through comparative proteomic analysis, it has been found that the expression abundances of some components of PSI (PsaF) and PSII (D1, LHCA, LHCB) detected in sto mutant decreased (Table 4). The results show that STO is necessary for the normal accumulation of photopigment protein complex. It is speculated that the rate of photosynthetic electrons in sto mutant might be decreased. It may be related to the need for plants to face high-energy UV-B light environment changes, forcing plants to sacrifice energy transfer rate in growth to cope with high light intensity stress for nonphotochemical quenching. High-energy UV-B radiation damages PSII and PSI, affects photosynthesis, and then affects life activities. The results of proteomics suggest that STO could maintain the normal structure of antenna protein complex under UV-B radiation, improve the photosynthetic electron transfer rate to meet the needs of survival, and adapt to UV-B radiation. Overexpression of STO under UV-B radiation significantly increases the NPQ level by detecting chlorophyll fluorescence parameters (Figure 10). STO maintains the accumulation of LHC family proteins under UV-B radiation and improves the heat dissipation ability of NPQ through comparative proteomic analysis (Figure 10). The UV-B response proteins dependent on STO are mainly located on thylakoid membrane and chloroplast membrane (Figures 4 and 5). Many LHC family proteins in sto mutant were down regulated after UV-B radiation (Table 4), and the light protection ability decreased. On the contrary, the NPQ ability of 35S-STO-GFP seedlings was enhanced, which could release the extra energy of UV-B and reduce the damage (Figure 10). Therefore, the interference of UV-B on NPQ (Figure 11) may be caused by the down regulation of LHC family protein. STO could stabilize the normal structure of light harvesting protein complex, perform photosynthesis, and maintain the normal metabolism of life activities by maintaining the accumulation of antenna protein complex, improving the NPQ ability, and releasing extra energy.

\subsection{STO Had Crosstalk with ABA upon UV-B Radiation}

The plant hormone ABA inhibits root elongation and seed germination. According to the proteomics data (Table 5), auxin and ABA signaling factors strongly associate with STO in a UV-B-dependent manner. In the sto mutant, the expression of auxin-related proteins (DRM2, AXR4) and negative regulatory protein ERD15 of ABA were up-regulated after UV-B radiation. The axr1-3 and ibr5-1 mutants grown on MS plates containing $10 \mu \mathrm{M}$ ABA had a similar root length, which was greater than that of the WT, suggesting that AXR1 promotes ABA signaling and inhibits root elongation [57]. We found that $A X R 4$ and $A B I 5$ were significantly up-regulated in the sto mutant under UV-B radiation (Table 5 and Figure 9), suggesting STO may attenuate ABA signaling and promote hypocotyl elongation by inhibiting AXR4 expression.

The accumulation and distribution of auxin in drm1-drm2-cmt3 mutants were affected [58]. DNA methylation is an epigenetic modification of DNA, which plays an important role in the development of embryo, organ, flower and fruit, and the response of plants to different stresses [59]. Mutations in DNA methyltransferase lead to DNA methylation loss, affect gene expression regulating auxin synthesis, transport and signaling pathways, and lead to developmental abnormalities [58]. DNA methyltransferase CMT3 and DRM2 were up-regulated to increase glutathione reductase (GR), ascorbic acid peroxidase (APX), and catalase (CAT) under $\mathrm{Cu}$ or $\mathrm{Cd}$ stress, which improve resistance to heavy metals in plants [60]. DRM2 protein expression was significantly up-regulated in $(+) s t o /(+) \mathrm{WT}$ and down-regulated in (+)sto/(+)WT (Table 5), suggesting that the DNA methylation degree of plants increases with STO mutation after UV-B radiation, which may maintain survival by sacrificing growth energy and improving plant resistance.

Transcription factors DREB1A/CBF3 and DREB2A interact specifically with cis-acting elements (DRE, CRT) related to cold and drought stress response gene expression in Arabidopsis. Overexpression of constitutive activity $D R E B 2 A$ enhances drought tolerance and regulates the expression of many 
water stress-induced genes [61]. DREB1A/2A in 35S-STO-GFP plants were significantly up-regulated after UV-B treatment (Figure 9).

The ERD (Early Responsive to Dehydration) genes can be rapidly induced upon abiotic stresses such as drought, low temperature, or salinity [62]. Belonging to a highly conserved family, ERD15 is not only a central component of several stress responses in Arabidopsis but also involved in stomatal closure. Previous studies have reported the sequence similarity between the light stress-regulated genes (Lsr1) and AtERD15, as well as the significant Lsr1 transcription inhibition by UV-A irradiation [63]. Now, the molecular mechanism of ERD15 in UV-B signaling is not known. Our results suggest that STO may repress ERD15 to enhance ABA signaling.

\section{Materials and Methods}

\subsection{Plant Materials and UV-B Treatment}

The seedlings of Arabidopsis thaliana wild-type Columbia (Col-0), sto mutant, and 35S::STO-GFP [15] were used. After surface sterilization in $30 \%(v / v)$ chlorine bleach (sodium hypochlorite) for $13 \mathrm{~min}$, the seeds were sown on 1/2 MS (half-strength Murashige and Skoog) medium containing $3 \%$ sucrose and $0.8 \%$ agar. The seeds went through a four-day stratification at $4{ }^{\circ} \mathrm{C}$ in the dark before grown in white light $\left(100 \mu \mathrm{mol} \mathrm{m}{ }^{-2} \mathrm{~s}^{-1}\right)$ for seven days (light/dark, $\left.16 / 8 \mathrm{~h}\right)$ at $21^{\circ} \mathrm{C}$. We performed the UV-B treatment in narrowband UV-B tubes (Philips TL 20W/01 RS) at $0.6 \mathrm{~W} \mathrm{~m}^{-2} \mathrm{~s}^{-1}$, with a radiation rate at approximately $0.785 \mu \mathrm{mol} \mathrm{m}^{-2} \mathrm{~s}^{-1}$. After UV-B radiation, the seedlings were grown under $10 \mu \mathrm{mol}$ $\mathrm{m}^{-2} \mathrm{~s}^{-1}$ white light for $4 \mathrm{~h}$. To filter UV-B and block UV-C, we used the cellulose acetate film (+UV-B). To block both UV-B and UV-C, we used the mylar film (-UV-B). The Quantithern Light Meter (Hansatec) was used to measure light intensity.

\subsection{Protein Extraction and Digestion}

For protein lysis, $300 \mathrm{mg}$ randomly selected samples from WT and sto mutant seedlings exposed to UV-B were ground with liquid nitrogen and homogenized in SDT (4\% SDS, $100 \mathrm{mM}$ Tris- $\mathrm{HCl}$, $1 \mathrm{mM}$ DTT, pH7.6) buffer. The BCA Protein Assay Kit (Bio-Rad) was used to quantify the proteins. Trypsin was used to digest the proteins into peptides according to Matthias Mann [64]. After digestion, the samples went through desalting using the C18 Cartridges (standard density, bed I.D. $7 \mathrm{~mm}, 3 \mathrm{~mL}$, Sigma) and were concentrated by vacuum centrifugation and reconstitution in $40 \mu \mathrm{L}$ dissolution buffer. A280 was measured to quantify the peptides.

\section{3. iTRAQ Labeling, Strong Cation Exchange (SCX) Fractionation, and LC-MS/MS Analysis}

A $100 \mu \mathrm{g}$ peptide mixture of each sample was labeled with the iTRAQ reagent according to the manufacturer's instructions (Applied Biosystems). The labeled samples were designated (-)UV-B-Col/114, (+)UV-B-Col/115, (-)UV-B-sto/116, and (+)UV-B-sto/117 (sample/isobaric tag). We prepared three replicates for each sample. We used SCX chromatography to fractionate each labeled sample into 15 parts before desalting and lyophilization [65]. The resulting fractionated samples were separated on an Easy nLC (nanoliter flow rate) HPLC system (Thermo Scientific). The samples were chromatographed and subjected to $1 \mathrm{~h}$ gradient Q-Exactive analysis 45 times as described previously [65].

\subsection{Protein Characterization}

After obtaining the MS/MS data, we used MASCOT v2.3.02 (Matrix Science, Berkshire, UK) to identify and quantify the proteins using Proteome Discoverer 1.4. Before the protein search, we set the enzyme as trypsin, MS/MS fragment ion mass tolerance at $\pm 0.1 \mathrm{Da}$, peptide mass tolerance at $\pm 20 \mathrm{ppm}$, missed cleavages at 2, fixed modifications as carbamidomethylation (Cys) and iTRAQ 4 plex (N-terminal and Lys), and variable modifications as oxidation (Met) and iTRAQ 4plex (Tyr). The TAIR10_pep_20101214.fasta database was used. The maximum false discovery rate (FDR) of 
the data was $5 \%$ to be eligible for quantification. For protein ratio calculation, we employed the median of unique peptides, normalizing all peptide ratios by the median (normalized to 1 prior to normalization of all peptide ratios). We also verified the repeatability of the replicates by comparing the protein abundance of each biological replicate to 1 . Proteins with an expression level change of $>1.2$ (up-regulated) or $<0.83$ (down-regulated) were considered DEPs; $P$-value $<0.05$.

\subsection{Function Annotation}

The proteomics data were further processed using Clustvis (https://biit.cs.ut.ee/clustvis/). The DEPs were functionally annotated using Blast2GO [66] and were mapped for KEGG pathways. The protein ID number of the target protein was converted to the KO (KEGG Orthology) number through Uniprot website. KEGG paththway annotation was completed through the KEGG online website (https://www.kegg.jp/) [67]. The distribution of the KEGG pathway in the target protein set and identified protein set was analyzed by Fisher's exact test. The interaction between the target proteins can be analyzed through the STRING (http://string-db.org/) database. The interacting network was generated by CytoScape software (version number: 3.2.1, http://www.cytoscape.org/).

\subsection{Quantitative Real-Time PCR}

Total RNA was extracted using RNAiso Plus (\#9108, TaKaRa, Dalian, China), and cDNA was synthesized using PrimeScript ${ }^{\mathrm{TM}}$ RT reagent Kit with gDNA Eraser (\#RR047A, TaKaRa, Dalian, China) for qRT-PCR reactions on the QuantStudio 6 Flex Real-Time PCR System (Applied Biosystems, Foster City, USA). We used LunaßUniversal qPCR Master Mix (\#M3003L, NEB, Massachusetts, USA) for the reactions and Actin 2 as the internal reference. Specific Primer 5.0 software was used to design the primers for the experiment (Table 7). Three technical replicates and three biological replicates were analyzed.

Table 7. The primers of qRT-PCR.

\begin{tabular}{ccc}
\hline Name & Primer Name & Sequence \\
\hline Actin & ACTIN2-F & GTTGGGATGAACCAGAAGGA \\
& ACTIN2-R & GCTCTTCAGGAGCAATACGAAG \\
PAL1 & PAL1-QF & GATTATGGATTCAAGGGAG \\
& PAL1-QR & TTTGCGAGACGAGATTAG \\
FLS & FLS-QF & ATACAGGGAGGTGAATGAA \\
& FLS-QR & ACACGGCGGATAATAGTT \\
CHS & CHS-QF & ACGTCACGTGTTGAGCGAGTATGG \\
& CHS-QR & GAGGAACGCTGTGCAAGACGACTG \\
LHCA3 & LHCA3-QF & TTCTTCACTTACCTCCTCTG \\
& LHCA3-QR & GTCTGTTGGCTCCTTGCT \\
LHCA2 & LHCA2-QF & TAGCCTCCCTGGTGACTT \\
& LHCA2-QR & GGATTCCGATCTTCGTTAG \\
PSAD-2 & PSAD-2-QF & GCCATAACAACCACTACTTC \\
& PSAD-2-QR & ACTGGAGCTTCTTTCACG \\
DREB2A & DREB2A-QF & TGAAAGGTAAAGGAGGAC \\
& DREB2A-QR & CCAAAGGACCATACATAGC \\
DREB1A & DREB1A-QF & GTGGGTTTGTGAGGTTAGAG \\
& DREB1A-QR & CCTTAGCGCAAGTTGATT \\
RD22 & RD22-QF & CCAAACACTCCCATTCCC \\
& RD22-QR & ACACCTCCCTTTCCAACG \\
ABI5 & ABI5-QF & GGTGAGAATCATCCGTTTA \\
& ABI5-QR & TCCTCTGCGTTCCAAATA \\
P5CS1 & P5CS1-QF & AGGGAAAGTTCCAGAAAG \\
& P5CS1-QR & CATAACTAAGCGAGCCAC \\
& SAD2-QF & CTTATGACCGACAGAAACA \\
& SAD2-QR & CAACAGTGAGACGCAGAT \\
\hline
\end{tabular}




\subsection{Parallel Reaction Monitoring (PRM) Analysis}

We validated the iTRAQ data using LC-PRM/MS (Shanghai Applied Protein Technology, Shanghai, China) [68]. In brief, we followed the instructions of iTRAQ for peptide preparation, $10 \mathrm{fmol}$ heavy isotope-labeled peptide fragment DSPSAPVNVTVR (framed V: a heavy isotope label synthesized by GL Biochem) was added into each sample as the internal standard. An Easy nLC-1200 system (Thermo Scientific, MA, USA) was used for separating the tryptic peptides. The $1 \mathrm{~h}$ liquid chromatography had acetonitrile gradients ranging from $5 \%$ to $35 \%$ over $45 \mathrm{~min}$. The Q Exactive Plus mass spectrometer (Thermo Scientific) was used in the positive ion mode for a full MS1 scan with a resolution of 60,000 at $200 \mathrm{~m} / \mathrm{z}$, an automatic gain control (AGC) target value of $3.0 \times 10^{-6}$, and a $200 \mathrm{~ms}$ maximum ion injection time. After the full MS scans, 20 PRM scans were run with the same parameters, except for the resolution and maximum ion injection time, which were adjusted to 30,000 and $120 \mathrm{ms,}$ respectively. A 1.6 Th window was set for isolating the target peptides. Normalized collision energy of $27 \mathrm{eV}$ in a higher-energy dissociation (HCD) collision cell was set for ion activation/dissociation. Skyline was used for raw data analysis to quantify the signal intensity of each individual peptide sequence whose abundance changed significantly. After the quantification, the data were normalized to the standard reference. Each sample had three biological replicates.

\subsection{Flavonoid and Anthocyanin Measurement}

To measure flavonoid contents, $0.1 \mathrm{~g}$ of 12-day-old seedlings exposed to UV-B were quantified according to Kucera [69]. The measurement of anthocyanin levels for $0.1 \mathrm{~g}$ of 12-day-old seedlings exposed to UV-B had been described previously [70,71].

\subsection{Photochemical Activity Measurement}

We measured the chlorophyll fluorescence parameters, including the maximal PSII efficiency $(\mathrm{Fv} / \mathrm{Fm})$ and non-photochemical quenching (NPQ), using Chlorophyll Fluorescence Imager (Technologica, Colchester, UK). The samples were put in the dark for $20 \mathrm{~min}$ before measurements [72].

\subsection{Measurements for IAA, ABA and $J A$}

We measured the IAA, ABA, and JA content in each sample. Two hundred milligrams of each liquid nitrogen-frozen fresh sample was homogenized in the TissueLyser (QIAGEN, Dusseldorf, Germany), then send to Hunan Provincial Key Laboratory of Phytohormones (Hunan Agricultural University, Changsha, China) for further extract and measurements according to Zhou [73]. Each sample had three biological replicates.

\subsection{Data Analysis}

GraphPad Prism 7.0 software was used for statistical analysis and drawing. The statistical analysis includes One-Way ANOVA analysis, Dunnett's multiple tests and difference significance analysis. Differences were considered significant for $P<0.05$. Adobe Photoshop CC was used for image processing, and Adobe Illustrator CC was used for chart layout.

\section{Conclusions}

The putative mechanism is schemed (Figure 12) to depict the involvement of STO in UV-B signaling. UV-B is received by the photoreceptor UVR8 to induce STO accumulation in plants. STO promotes the accumulation the PSBs and LHCs to stabilize the antenna system and release excess energy for protecting the photosynthetic machinery. Meanwhile, STO represses the accumulation of UGT84A2 to decrease anthocyanin synthesis and promotes the accumulation of FLS to increase flavonoid synthesis for photoprotection. UV-B increases flavonoid and anthocyanin synthesis, but does not depend on STO. 


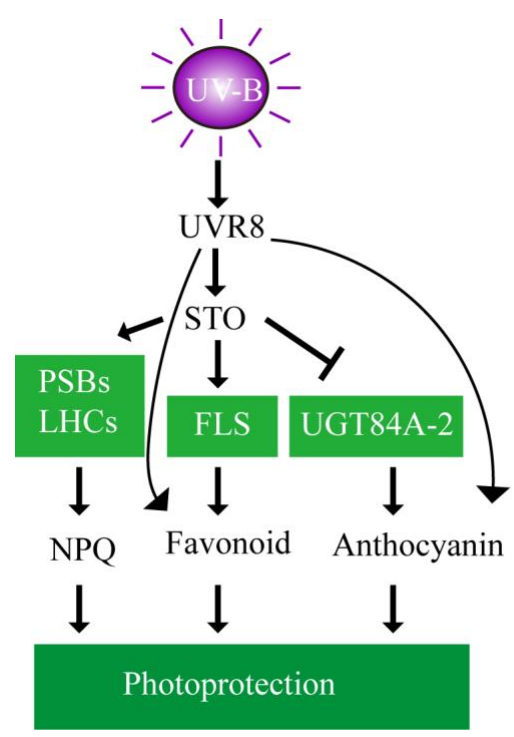

Figure 12. Schematic model of photoprotection mediated by STO in the presence of UV-B radiation. The putative mechanisms are schemed to depict the involvement of STO in UV-B signaling. UV-B is received by the photoreceptor UVR8 to induce STO accumulation in plants. STO protects the photosynthetic machinery by stabilizing the antenna system and releasing excess energy. Meanwhile, STO represses anthocyanin synthesis and promotes flavonoid synthesis for photoprotection.

Author Contributions: S.L. designed the project. G.L. performed most of the experiments, and D.L. and H.X. participated in the experiments. G.L., D.L., and C.N. analyzed the iTRAQ data. L.X. and J.T. measured the plant hormones. G.L. and D.L. wrote the draft of the manuscript, and S.L. revised the draft. Funding acquisition and project administration were done by S.L. All authors have read and approved the submitted vision of the manuscript.

Funding: This work was supported by the National Natural Science Foundation of China (Grant No. 31670266 and 31570372), the Natural Science Foundation of Guangdong Province, China (Grant No. 2017A030313115 and 2017A030310168), the Innovation Project of Graduate School of South China Normal University (20161kxm10), and the Leading Scientists Project of Guangdong Province and the Guangdong Pearl River Scholar Funded Scheme (2012).

Acknowledgments: We thank the Shanghai Applied Protein Technology Co., Ltd., for technical support in the iTRAQ and PRM analysis. We thank Professor Lars Olof Björn from Lund University, Sweden, for the guidance and providing the UV filters.

Conflicts of Interest: The authors declare no conflicts of interest.

\section{Abbreviations}

$\begin{array}{ll}\text { STO } & \text { Salt Tolerance } \\ \text { HY5 } & \text { Elongated Hypocotyl 5 } \\ \text { iTRAQ } & \text { Isobaric Tags for Relative and Absolute Quantification } \\ \text { PRM } & \text { Parallel Reaction Monitoring } \\ \text { GO } & \text { Gene Ontology } \\ \text { KEGG } & \text { Kyoto Encyclopedia of Genes and Genomes } \\ \text { UVR8 } & \text { UV Resistance Locus } 8 \\ \text { COP1 } & \text { Constitutively Photomorphogenic 1 } \\ \text { RUPs } & \text { Repressor of UV-B Photomorphogenesis } \\ \text { PIF4 } & \text { Phytochrome Interacting Factor 4 } \\ \text { PAL } & \text { Phenylalanine Ammonia Lyase } \\ \text { CHS } & \text { Chalcone Synthase } \\ \text { DFR } & \text { Dihydroflavonol Reductase } \\ \text { FLS } & \text { Flavonol Synthase }\end{array}$




\section{References}

1. Oravecz, A.; Baumann, A.; Máté, Z.; Brzezinska, A.; Molinier, J.; Oakeley, E.J.; Ádám, É.; Schäfer, E.; Nagy, F.; Ulm, R. CONSTITUTIVELY PHOTOMORPHOGENIC1 is required for the UV-B response in Arabidopsis. Plant Cell 2006, 18, 1975-1990. [CrossRef] [PubMed]

2. Favory, J.J.; Stec, A.; Gruber, H.; Rizzini, L.; Oravecz, A.; Funk, M.; Albert, A.; Cloix, C.; Jenkins, G.I.; Oakeley, E.J. Interaction of COP1 and UVR8 regulates UV-B-induced photomorphogenesis and stress acclimation in Arabidopsis. EMBO J. 2009, 28, 591-601. [CrossRef]

3. Li, F.; Sun, J.; Wang, D.; Bai, S.; Clarke, A.K.; Holm, M. The B-box family gene STO (BBX24) in Arabidopsis thaliana regulates flowering time in different pathways. PLoS ONE 2014, 9, e87544. [CrossRef] [PubMed]

4. Ulm, R.; Jenkins, G.I. Q \& A: How do plants sense and respond to UV-B radiation? BMC Biol. 2015, 13, 45-50.

5. Biever, J.J.; Gardner, G. The relationship between multiple UV-B perception mechanisms and DNA repair pathways in plants. Environ. Exp. Bot. 2016, 124, 89-99. [CrossRef]

6. Jenkins, G.I. Structure and function of the UV-B photoreceptor UVR8. Curr. Opin. Struct. Biol. 2014, 29, 52-57. [CrossRef]

7. Dotto, M.; Gómez, M.S.; Soto, M.S.; Casati, P. UV-B radiation delays flowering time through changes in the PRC2 complex activity and miR156 levels in Arabidopsis thaliana. Plant Cell Environ. 2018, 41, 1394-1406. [CrossRef]

8. Rizzini, L.; Favory, J.J.; Cloix, C.; Faggionato, D.; O’Hara, A.; Kaiserli, E.; Baumeister, R.; Schäfer, E.; Nagy, F.; Jenkins, G.I. Perception of UV-B by the Arabidopsis UVR8 protein. Science 2011, 332, 103-106. [CrossRef] [PubMed]

9. Wu, D.; Hu, Q.; Yan, Z.; Chen, W.; Yan, C.; Huang, X.; Zhang, J.; Yang, P.; Deng, H.; Wang, J. Structural basis of ultraviolet-B perception by UVR8. Nature 2012, 484, 214-219. [PubMed]

10. Fernández, M.B.; Tossi, V.; Lamattina, L.; Cassia, R. A comprehensive phylogeny reveals functional conservation of the UV-B photoreceptor UVR8 from green algae to higher plants. Front. Plant Sci. 2016, 7, 1698-1703. [CrossRef]

11. Liang, T.; Yang, Y.; Liu, H. Signal transduction mediated by the plant UV-B photoreceptor UVR8. New Phytol. 2019, 221, 1247-1252. [CrossRef]

12. Binkert, M.; Kozma-Bognár, L.; Terecskei, K.; De Veylder, L.; Nagy, F.; Ulm, R. UV-B-responsive association of the Arabidopsis bZIP transcription factor ELONGATED HYPOCOTYL5 with target genes, including its own promoter. Plant Cell 2014, 26, 4200-4213. [CrossRef]

13. Yang, Y.; Liang, T.; Zhang, L.; Shao, K.; Gu, X.; Shang, R.; Shi, N.; Li, X.; Zhang, P.; Liu, H. UVR8 interacts with WRKY36 to regulate HY5 transcription and hypocotyl elongation in Arabidopsis. Nat. Plants 2018, 4, 98-107. [CrossRef]

14. Liang, T.; Mei, S.; Shi, C.; Yang, Y.; Peng, Y.; Ma, L.; Wang, F.; Li, X.; Huang, X.; Yin, Y. UVR8 interacts with BES1 and BIM1 to regulate transcription and photomorphogenesis in Arabidopsis. Dev. Cell 2018, 44, 512-523.e5. [CrossRef]

15. Jiang, L.; Wang, Y.; Li, Q.F.; Björn, L.O.; He, J.X.; Li, S.S. Arabidopsis STO/BBX24 negatively regulates UV-B signaling by interacting with COP1 and repressing HY5 transcriptional activity. Cell Res. 2012, 22, 1046-1057. [CrossRef]

16. Nagaoka, S.; Takano, T. Salt tolerance-related protein STO binds to a Myb transcription factor homologue and confers salt tolerance in Arabidopsis. J. Exp. Bot. 2003, 54, 2231-2237. [CrossRef]

17. Hayes, S.; Velanis, C.N.; Jenkins, G.I.; Franklin, K.A. UV-B detected by the UVR8 photoreceptor antagonizes auxin signaling and plant shade avoidance. Proc. Natl. Acad. Sci. USA 2014, 111, 11894-11899. [CrossRef] [PubMed]

18. Gangappa, S.N.; Crocco, C.D.; Henrik, J.; Sourav, D.; Chamari, H.; Magnus, H.; Botto, J.F. The Arabidopsis B-BOX protein BBX25 interacts with HY5, negatively regulating BBX22 expression to suppress seedling photomorphogenesis. Plant Cell 2013, 25, 1243-1257. [CrossRef] [PubMed]

19. Job, N.; Yadukrishnan, P.; Bursch, K.; Datta, S.; Johansson, H. Two B-box proteins regulate photomorphogenesis by oppositely modulating HY5 through their diverse C-terminal domains. Plant Physiol. 2018, 176, 2963-2976. [CrossRef] [PubMed]

20. Lyu, G.; Li, D.; Li, S.; Hu, H. STO and GA negatively regulate UV-B-induced Arabidopsis root growth inhibition. Plant Signal. Behav. 2019. [CrossRef] 
21. Chen, C.; Xia, R.; Chen, H.; He, Y. TBtools, a Toolkit for Biologists integrating various HTS-data handling tools with a user-friendly interface. Biorxiv 2018, 289660. [CrossRef]

22. Imtiaz, M.; Yang, Y.; Liu, R.; Xu, Y.; Khan, M.A.; Wei, Q.; Gao, J.; Hong, B. Identification and functional characterization of the BBX24 promoter and gene from chrysanthemum in Arabidopsis. Plant Mol. Biol. 2015, 89, 1-19. [CrossRef] [PubMed]

23. Huang, X.; Ouyang, X.; Yang, P.; Lau, O.S.; Chen, L.; Wei, N.; Deng, X.W. Conversion from CUL4-based COP1-SPA E3 apparatus to UVR8-COP1-SPA complexes underlies a distinct biochemical function of COP1 under UV-B. Proc. Natl. Acad. Sci. USA 2013, 110, 16669-16674. [CrossRef] [PubMed]

24. Gruber, H.; Heijde, M.; Heller, W.; Albert, A.; Seidlitz, H.K.; Ulm, R. Negative feedback regulation of UV-B-induced photomorphogenesis and stress acclimation in Arabidopsis. Proc. Natl. Acad. Sci. USA 2010, 107, 20132-20137. [CrossRef]

25. Treutter, D. Significance of flavonoids in plant resistance and enhancement of their biosynthesis. Plant Biol. 2005, 7, 581-591. [CrossRef]

26. Choudhary, K.K.; Agrawal, S.B. Cultivar specificity of tropical mung bean (Vigna radiata L.) to elevated ultraviolet-B: Changes in antioxidative defense system, nitrogen metabolism and accumulation of jasmonic and salicylic acids. Environ. Exp. Bot. 2014, 99, 122-132. [CrossRef]

27. Liu, X.; Li, Q.; Yue, M.; Zhang, X.; Zhang, R.; Zhang, B.; Wang, M. Nitric oxide is involved in integration of UV-B absorbing compounds among parts of clonal plants under a heterogeneous UV-B environment. Physiol. Plant. 2015, 155, 180-191. [CrossRef]

28. Hideg, É.; Jansen, M.A.; Strid, Å. UV-B exposure, ROS, and stress: Inseparable companions or loosely linked associates? Trends Plant Sci. 2013, 18, 107-115. [CrossRef]

29. Bais, A.F.; Lucas, R.M.; Bornman, J.F.; Williamson, C.E.; Sulzberger, B.; Austin, A.T.; Wilson, S.R.; Andrady, A.L.; Bernhard, G.; McKenzie, R.L. Environmental effects of ozone depletion, UV radiation and interactions with climate change: UNEP Environmental Effects Assessment Panel, update 2017. Photochem. Photobiol. Sci. 2018, 17, 127-179. [CrossRef]

30. Morales, L.O.; Brosché, M.; Vainonen, J.; Jenkins, G.I.; Wargent, J.J.; Sipari, N.; Strid, Å.; Lindfors, A.V.; Tegelberg, R.; Aphalo, P.J. Multiple roles for UV RESISTANCE LOCUS8 in regulating gene expression and metabolite accumulation in Arabidopsis under solar ultraviolet radiation. Plant Physiol. 2013, 161, 744-759. [CrossRef]

31. Zhang, X.; Abrahan, C.; Colquhoun, T.A.; Liu, C. A proteolytic regulator controlling chalcone synthase stability and flavonoid biosynthesis in Arabidopsis. Plant Cell 2017, 29, 1157-1174. [CrossRef] [PubMed]

32. Wang, N.; Jiang, S.; Zhang, Z.; Fang, H.; Xu, H.; Wang, Y.; Chen, X. Malus sieversii: The origin, flavonoid synthesis mechanism, and breeding of red-skinned and red-fleshed apples. Hortic. Res. 2018, 5, 70-81. [CrossRef] [PubMed]

33. Maier, A.; Schrader, A.; Kokkelink, L.; Falke, C.; Welter, B.; Iniesto, E.; Rubio, V.; Uhrig, J.F.; Hülskamp, M.; Hoecker, U. Light and the E3 ubiquitin ligase COP 1/SPA control the protein stability of the MYB transcription factors PAP 1 and PAP 2 involved in anthocyanin accumulation in Arabidopsis. Plant J. 2013, 74, 638-651. [CrossRef] [PubMed]

34. Qiu, Z.; Wang, H.; Li, D.; Yu, B.; Hui, Q.; Yan, S.; Huang, Z.; Cui, X.; Cao, B. Identification of candidate HY5-dependent and-independent regulators of anthocyanin biosynthesis in Tomato. Plant Cell Physiol. 2018, 60, 643-656. [CrossRef]

35. Ren, H.; Han, J.; Yang, P.; Mao, W.; Liu, X.; Qiu, L.; Qian, C.; Liu, Y.; Chen, Z.; Ouyang, X.; et al. Two E3 ligases antagonistically regulate the UV-B response in Arabidopsis. Proc. Natl. Acad. Sci. USA 2019, 116, 4722-4731. [CrossRef]

36. Stracke, R.; FAVORY, J.J.; Gruber, H.; Bartelniewoehner, L.; Bartels, S.; Binkert, M.; Funk, M.; Weisshaar, B.; Ulm, R. The Arabidopsis bZIP transcription factor HY5 regulates expression of the PFG1/MYB12 gene in response to light and ultraviolet-B radiation. Plant Cell Environ. 2010, 33, 88-103.

37. Gou, J.Y.; Felippes, F.F.; Liu, C.J.; Weigel, D.; Wang, J.W. Negative regulation of anthocyanin biosynthesis in Arabidopsis by a miR156-targeted SPL transcription factor. Plant Cell 2011, 23, 1512-1522. [CrossRef]

38. Ramsay, N.A.; Glover, B.J. MYB-bHLH-WD40 protein complex and the evolution of cellular diversity. Trends Plant Sci. 2005, 10, 63-70. [CrossRef]

39. Tanaka, Y.; Ohmiya, A. Seeing is believing: Engineering anthocyanin and carotenoid biosynthetic pathways. Curr. Opin. Biotechnol. 2008, 19, 190-197. [CrossRef] 
40. Jaakola, L. New insights into the regulation of anthocyanin biosynthesis in fruits. Trends Plant Sci. 2013, 18, 477-483. [CrossRef]

41. Shin, D.H.; Choi, M.G.; Kim, K.; Bang, G.; Cho, M.; Choi, S.B.; Choi, G.; Park, Y.-I. HY5 regulates anthocyanin biosynthesis by inducing the transcriptional activation of the MYB75/PAP1 transcription factor in Arabidopsis. FEBS Lett. 2013, 587, 1543-1547. [CrossRef] [PubMed]

42. Yang, F.; Cai, J.; Yang, Y.; Liu, Z. Overexpression of microRNA828 reduces anthocyanin accumulation in Arabidopsis. Plant Cell Tissue Organ Cult. (PCTOC) 2013, 115, 159-167. [CrossRef]

43. Qin, X.; Pi, X.; Wang, W.; Han, G.; Zhu, L.; Liu, M.; Cheng, L.; Shen, J.-R.; Kuang, T.; Sui, S.-F. Structure of a green algal photosystem I in complex with a large number of light-harvesting complex I subunits. Nat. Plants 2019, 5, 263-272. [CrossRef]

44. Yu, G.; Li, W.; Yuan, Z.; Cui, H.; Lv, C.; Gao, Z.; Han, B.; Gong, Y.; Chen, G. The effects of enhanced UV-B radiation on photosynthetic and biochemical activities in super-high-yield hybrid rice Liangyoupeijiu at the reproductive stage. Photosynthetica 2013, 51, 33-44. [CrossRef]

45. Shi, S.B.; Zhu, Z.W.Y.; Li, H.M.; Zhou, D.W.; Fa, H.; Zhao, X.Q.; Tang, Y.H. Photosynthesis of Saussurea superba and Gentiana straminea is not reduced after long-term enhancement of UV-B radiation. Environ. Exp. Bot. 2004, 51, 75-83. [CrossRef]

46. Kataria, S.; Jajoo, A.; Guruprasad, K.N. Impact of increasing Ultraviolet-B (UV-B) radiation on photosynthetic processes. J. Photochem. Photobiol. B Biol. 2014, 137, 55-66. [CrossRef]

47. $\mathrm{Xu}, \mathrm{C}$;; Sullivan, J.H. Reviewing the technical designs for experiments with ultraviolet-B radiation and impact on photosynthesis, DNA and secondary metabolism. J. Integr. Plant Biol. 2010, 52, 377-387. [CrossRef]

48. Allorent, G.; Lefebvre-Legendre, L.; Chappuis, R.; Kuntz, M.; Truong, T.B.; Niyogi, K.K.; Ulm, R.; Goldschmidt-Clermont, M. UV-B photoreceptor-mediated protection of the photosynthetic machinery in Chlamydomonas reinhardtii. Proc. Natl. Acad. Sci. USA 2016, 113, 14864-14869. [CrossRef]

49. Külheim, C.; Ågren, J.; Jansson, S. Rapid regulation of light harvesting and plant fitness in the field. Science 2002, 297, 91-93. [CrossRef]

50. Funk, C.; Schroeder, W.P.; Napiwotzki, A.; Tjus, S.E.; Renger, G.; Andersson, B. The PSII-S protein of higher plants: A new type of pigment-binding protein. Biochemistry 1995, 34, 11133-11141. [CrossRef]

51. Müller, P.; Li, X.P.; Niyogi, K.K. Non-photochemical quenching. A response to excess light energy. Plant Physiol. 2001, 125, 1558-1566. [CrossRef]

52. Bečková, M.; Yu, J.; Krynická, V.; Kozlo, A.; Shao, S.; Koník, P.; Komenda, J.; Murray, J.W.; Nixon, P.J. Structure of Psb29/Thf1 and its association with the FtsH protease complex involved in photosystem II repair in cyanobacteria. Philos. Trans. R. Soc. B Biol. Sci. 2017, 372, 20160394. [CrossRef]

53. Alboresi, A.; Ballottari, M.; Hienerwadel, R.; Giacometti, G.M.; Morosinotto, T. Antenna complexes protect photosystem I from photoinhibition. BMC Plant Biol. 2009, 9, 71-80. [CrossRef]

54. Hieber, A.D.; Bugos, R.C.; Yamamoto, H.Y. Plant lipocalins: Violaxanthin de-epoxidase and zeaxanthin epoxidase. Biochim. Biophys. Acta (BBA) Protein Struct. Mol. Enzymol. 2000, 1482, 84-91. [CrossRef]

55. Goss, R.; Lepetit, B. Biodiversity of NPQ. J. Plant Physiol. 2015, 172, 13-32. [CrossRef]

56. Schaller, S.; Wilhelm, C.; Strzałka, K.; Goss, R. Investigating the interaction between the violaxanthin cycle enzyme zeaxanthin epoxidase and the thylakoid membrane. J. Photochem. Photobiol. B Biol. 2012, 114, 119-125. [CrossRef]

57. Thole, J.M.; Beisner, E.R.; Liu, J.; Venkova, S.V.; Strader, L.C. Abscisic acid regulates root elongation through the activities of auxin and ethylene in Arabidopsis thaliana. G3 Genes Genomes Genet. 2014, 4, 1259-1274. [CrossRef]

58. Forgione, I.; Wołoszyńska, M.; Pacenza, M.; Chiappetta, A.; Greco, M.; Araniti, F.; Abenavoli, M.R.; Van Lijsebettens, M.; Bitonti, M.B.; Bruno, L. Hypomethylated drm1 drm2 cmt3 mutant phenotype of Arabidopsis thaliana is related to auxin pathway impairment. Plant Sci. 2019, 280, 383-396. [CrossRef]

59. Ronemus, M.J.; Galbiati, M.; Ticknor, C.; Chen, J.; Dellaporta, S.L. Demethylation-induced developmental pleiotropy in Arabidopsis. Science 1996, 273, 654-657. [CrossRef]

60. Greco, M.; Sáez, C.A.; Contreras, R.A.; Rodríguez-Rojas, F.; Bitonti, M.B.; Brown, M.T. Cadmium and/or copper excess induce interdependent metal accumulation, DNA methylation, induction of metal chelators and antioxidant defences in the seagrass Zostera marina. Chemosphere 2019, 224, 111-119. [CrossRef] 
61. Sakuma, Y.; Maruyama, K.; Osakabe, Y.; Qin, F.; Seki, M.; Shinozaki, K.; Yamaguchi-Shinozaki, K. Functional analysis of an Arabidopsis transcription factor, DREB2A, involved in drought-responsive gene expression. Plant Cell 2006, 18, 1292-1309. [CrossRef]

62. Yu, D.; Zhang, L.; Zhao, K.; Niu, R.; Zhai, H.; Zhang, J. VaERD15, a transcription factor gene associated with cold-tolerance in Chinese Wild Vitis amurensis. Front. Plant Sci. 2017, 8, 297. [CrossRef]

63. Dunaeva, M.; Adamska, I. Identification of genes expressed in response to light stress in leaves of Arabidopsis thaliana using RNA differential display. Eur. J. Biochem. 2001, 268, 5521-5529. [CrossRef]

64. Wiśniewski, J.R.; Zougman, A.; Nagaraj, N.; Mann, M. Universal sample preparation method for proteome analysis. Nat. Methods 2009, 6, 359-362. [CrossRef]

65. Li, M.; Zhang, K.; Long, R.; Sun, Y.; Kang, J.; Zhang, T.; Cao, S. iTRAQ-based comparative proteomic analysis reveals tissue-specific and novel early-stage molecular mechanisms of salt stress response in Carex Rigescens. Environ. Exp. Bot. 2017, 143, 99-114. [CrossRef]

66. Götz, S.; García-Gómez, J.M.; Terol, J.; Williams, T.D.; Nagaraj, S.H.; Nueda, M.J.; Robles, M.; Talón, M.; Dopazo, J.; Conesa, A. High-throughput functional annotation and data mining with the Blast2GO suite. Nucleic Acids Res. 2008, 36, 3420-3435. [CrossRef]

67. Minoru, K.; Susumu, G.; Yoko, S.; Miho, F.; Mao, T. KEGG for integration and interpretation of large-scale molecular data sets. Nucleic Acids Res. 2011, 40, D109-D114.

68. Peterson, A.C.; Russell, J.D.; Bailey, D.J.; Westphall, M.S.; Coon, J.J. Parallel reaction monitoring for high resolution and high mass accuracy quantitative, targeted proteomics. Mol. Cell. Proteom. 2012, 11, 1475-1488. [CrossRef]

69. Kucera, B.; Leubner-Metzger, G.; Wellmann, E. Distinct ultraviolet-signaling pathways in bean leaves. DNA damage is associated with $\beta-1,3$-glucanase gene induction, but not with flavonoid formation. Plant Physiol. 2003, 133, 1445-1452. [CrossRef]

70. Weiss, D.; Halevy, A.H. Stamens and gibberellin in the regulation of corolla pigmentation and growth in Petunia hybrida. Planta 1989, 179, 89-96. [CrossRef]

71. Meng, X.; Wang, X. Regulation of flower development and anthocyanin accumulation in Gerbera hybrida. J. Hortic. Sci. Biotechnol. 2004, 79, 131-137. [CrossRef]

72. Sedej, T.T.; Gaberščik, A. The effects of enhanced UV-B radiation on physiological activity and growth of Norway spruce planted outdoors over 5 years. Trees Struct. Funct. 2008, 22, 423-435. [CrossRef]

73. Zhou, L.; Xiao, L.; Xue, H. Dynamic cytology and transcriptional regulation of rice lamina joint development. Plant Physiol. 2017, 174, 1728-1746. [CrossRef] [PubMed]

(C) 2020 by the authors. Licensee MDPI, Basel, Switzerland. This article is an open access article distributed under the terms and conditions of the Creative Commons Attribution (CC BY) license (http://creativecommons.org/licenses/by/4.0/). 